\title{
Modelling apical constriction in epithelia using elastic shell theory
}

\author{
Gareth W. Jones · S. Jonathan Chapman
}

Received: date / Accepted: date

\begin{abstract}
Apical constriction is one of the fundamental mechanisms by which embryonic tissue is deformed, giving rise to the shape and form of the fully-developed organism. The mechanism involves a contraction of fibres embedded in the apical side of epithelial tissues, leading to an invagination or folding of the cell sheet. In this article the phenomenon is modelled mechanically by describing the epithelial sheet as an elastic shell, which contains a surface representing the continuous mesh formed from the embedded fibres. Allowing this mesh to contract, an enhanced shell theory is developed in which the stiffness and bending tensors of the shell are modified to include the fibres' stiffness, and in which the active effects of the contraction appear as body forces in the shell equilibrium equations. Numerical examples are presented at the end, including the bending of a plate and a cylindrical shell (modelling neurulation), and the invagination of a spherical shell (modelling simple gastrulation).
\end{abstract}

Keywords Apical constriction - Shell theory · Epithelial tissue $\cdot$ Elastic deformation

\section{Introduction}

The Earth is home to many millions of animal species. Despite the great variety in their shapes and sizes, each

G.W. Jones, S.J. Chapman

Oxford Centre for Industrial and Applied Mathematics,

Mathematical Institute,

24-29 St Giles',

Oxford,

England.

OX1 3LB

Tel.: +44 1865280618

E-mail: jonesg@maths.ox.ac.uk individual begins their life as a rapidly dividing ball of cells. Developmental biology is the field that describes and seeks to explain the growth and development of an organism from the one-cell stage to a fully developed individual. Within developmental biology, the important discipline of morphogenesis is primarily interested in the mechanisms which change the shape of the growing embryo, giving rise to the characteristic form of the animal. A full description of the developmental processes in an embryo is outside the scope of this article; we refer the interested reader to a textbook such as Gilbert (2006).

Most of the research into embryo growth is carried out with respect to model organisms, especially the tree frog Xenopus laevis due to the large size of its embryos. Many different stages have been identified in the developmental cycle (Davidson, 2008), among which one of the most important is gastrulation. This event fundamentally changes the topology of the embryo, setting up the main body plan of the organism (Stern, 2004). Different species employ different gastrulation patterns; however, these are comprised of relatively few basic tissue movements. In this article we will concentrate on only one of these characteristic movements, namely invagination. This process involves a sheet of cells (usually epithelial cells) bending inwards to form dimples, grooves or tubes. An archetypal example is given by early sea urchin gastrulation, where a single layer of epithelial cells bends inwards to form a dimple, which later extends to a tube known as the archenteron (Davidson et al, 1995). The effect of the invagination can be seen in Figure 1. A similar effect can be seen in Xenopus gastrulation, although the initial invaginating region is now a groove (the blastoporal groove) rather than a dimple (Keller et al, 2003). Neurulation is a further example of tissue invagination, whereby a sheet of epithelial tissue 
invaginates to form a groove, and eventually pinches off to form the neural tube, the precursor of the brain and spinal cord. A similar effect occurs in Drosophila melanogaster ventral furrow formation (Sweeton et al, 1991).

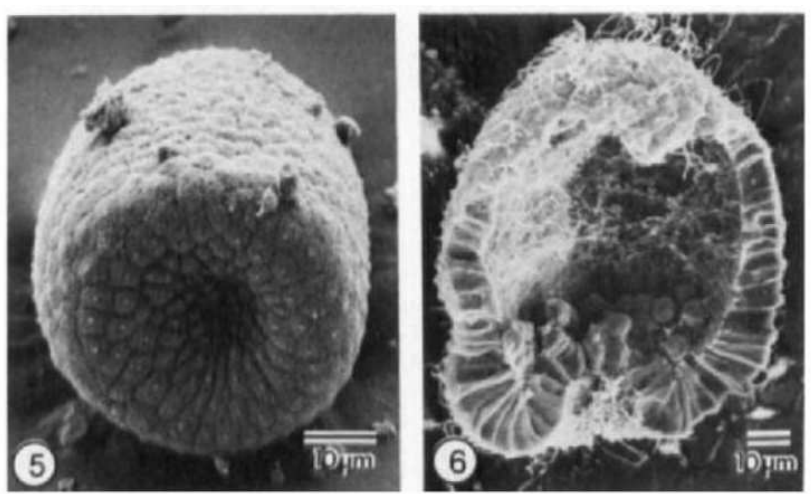

Figure 1 Invagination of a deciliated embryo of the sea urchin Lytechinus variegatus during mid-gastrula (left), and a crosssection through another embryo at the same stage of gastrulation (right). Reprinted from Morrill (1986) with permission from Elsevier

Invagination is an effect largely confined to epithelial tissue, which is one of the main tissue types in the animal kingdom. It is a sheet composed almost entirely of cells and has one main purpose: to serve as a barrier between two regions of different chemical composition (Wright and Alison, 1984). As a result epithelia form the lining of almost all tissues in the body, with a basal side in contact with the tissue and an apical side adjoining the medium in which the tissue is placed. Epithelia also form a large proportion of early embryos. The barrier property means that the cells must be tightly packed together, with occluding junctions near the apical side of the sheet preventing the passage of solutes. A diagram of a typical cuboidal epithelium is presented in Figure 2. Mechanical integrity is provided to the epithelium by a collection of anchoring junctions that attach the cells to one another through proteins which are embedded in the cell wall and connected to the cell cytoskeleton network. These junctions are concentrated in the upper half of the sheet, in a zone known as the junctional complex. Epithelia may be characterised according to their composition. If the cells are arranged in a single layer, the tissue is said to be simple; multi-layered epithelia are known as stratified. The second characteristic feature used to distinguish epithelia is the shape of the cells. Those epithelia consisting of cells which are wider than they are tall are squamous; if the cells are equally sized both vertically and laterally the epithelium is cuboidal; and finally epithelia with cells taller than they are wide are known as columnar epithelia. Epithelia in embryos, and hence those that are considered in this report, are generally either simple cuboidal or simple columnar epithelia.

Large-scale tissue movements in epithelial sheets are clearly dependent on the behaviour of the cells that comprise the tissue. The basic cell deformations that make up these tissue movements have been identified (Fristrom, 1988; Schöck and Perrimon, 2002; Pilot and Lecuit, 2005) and fall into one of a few elementary types: cell division and cell death; ingression or egression; cell intercalation; cell migration; and cell shape changes.

Changes in cell shape are accomplished by remodelling the cell cytoskeleton. Besides flattening, which expands the sheet and makes it thinner (and its opposite effect, columnarisation), and elongation along one planar axis (leading to anisotropic in-plane deformations), the deformation that we concentrate on in this article is apical constriction. In this process the apical surface of a cell contracts while the basal surface expands to accommodate the displaced cytoplasm. As the cells are still attached to each other, this motion results in an invagination of the tissue (see Figure 3 ). Biologically, the apical constriction process is increasingly well-understood (Pilot and Lecuit, 2005; Dawes-Hoang et al, 2005; Martin et al, 2009); the activation of certain gene pathways leads to the recruitment of myosin molecules to the mesh-like cortical cytoskeleton near the apical cell surface. This results in the contraction of the apical cell perimeter through the attachment of this cytoskeleton to the junctional complex. From the point of view of a single cell, the resultant deformation has been compared to the drawing of a purse-string. The cytoskeleton in the apical region of the cell can be thought of as being composed of two parts: a fine mesh of microfilaments and microtubules distributed throughout the entire apical cortex (particularly prevalent in cells possessing microvilli, where the structure is known as a terminal web), and a circumferential bundle of microfilaments which encircles the cell perimeter (Bement and Mooseker, 1996). The mechanism of apical constriction has been ascribed to contraction both of the cortical microfilaments (Dawes-Hoang et al, 2005; Lee and Harland, 2007) and of the circumferential bundle (Burnside, 1971; Thomas, 2001), and also to a combination of the two (Gordon and Brodland, 1987). Extension of the apical cytoskeleton has also been implicated in evagination (outward bending) of epithelia in vitro (Graebert et al, 1997). It should be noted that apical constriction is not the only mechanism that gives rise to invagination; columnarisation, and buckling due to in- 


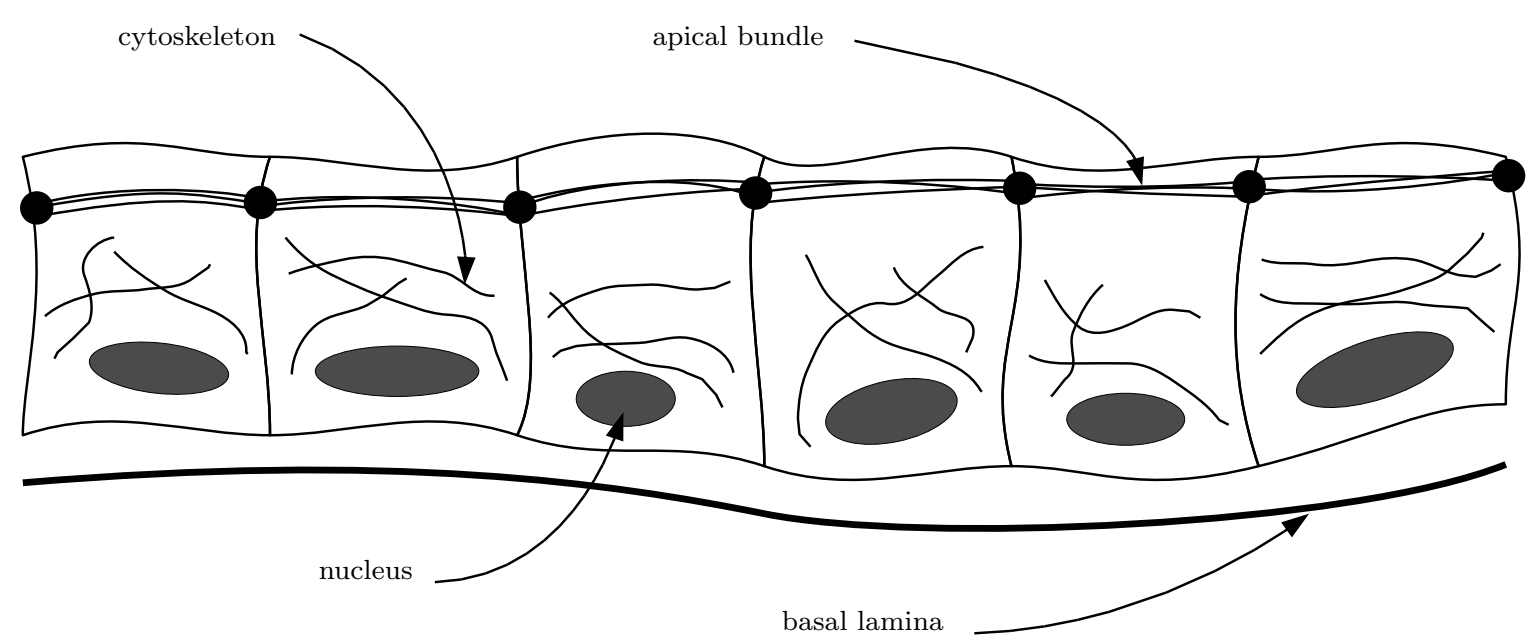

Figure 2 A diagram of a typical cuboidal epithelium.

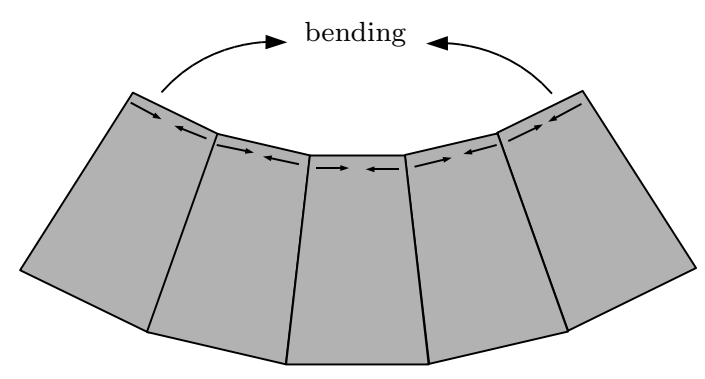

Figure 3 An illustration of how shortening the apices of epithelial cells can result in the bending of the tissue.

creased in-surface forces, can also play a role (Fristrom, 1988).

During the course of the last few decades, much progress has been made in this field by analysing the genetic and molecular pathways that govern the apical constriction process at a sub-cellular scale. However, morphogenesis is by its very nature a mechanical process, and an understanding of the mechanics is therefore an essential prerequisite for this important area of study (Hutson and Ma, 2008). Before the advent of computers and numerical simulations, the mechanical properties of embryos had been modelled by intricate mechanical machines. Lewis (1947) used such a contraption to simulate invagination in developing embryonic tissue. In time, the virtual cells represented as parts of such mechanisms were replaced by virtual cells in a computational framework, such as the well-known model of epithelial folding by Odell et al (1981). In this model the cells were modelled as a collection of viscoelastic struts, of which one per cell (i.e. the strut in the apical region) was allowed to contract, thus modelling apical constriction and the resultant tissue invagination.
Other early models treated the deforming tissue as a continuum material. In these formulations the positions and behaviours of the cells themselves are unimportant; it is the deformation of the tissue as a whole that is simulated. These models also exploit the thinness of the cell sheets being considered, assuming that the deformation of the sheet can be given in terms of its middle surface. Some of these models consider the tissue deformation to be caused by pre-existing patterns of certain diffusing chemicals (morphogens) and assume that the shape of the tissue is directly related to the concentrations of these chemicals, either phenomenologically (Cummings, 1990) or based on mechanical principles (Gierer, 1977). Mittenthal and co-workers (Mittenthal and Mazo, 1983; Mittenthal, 1987) developed a theory in which epithelial sheets were modelled as viscous fluids with respect to stretching deformations and as elastic shells under bending deformations. Hardin and Cheng (1986) used a fully elastic shell model to simulate the gastrulating sea urchin embryo, using finite element methods to determine the shape of the embryo. The mechanism of deformation, however, was not apical constriction but rather a tensile load applied to the inner shell surface. The model was later extended (Hardin and Keller, 1988) to the gastrulation of X. laevis; apical constriction in this model was simulated by the imposition of an external moment to the shell.

A further, more recent, class of continuum models for invagination do not consider the tissue to be thin, and treat the material as an elastic solid, whose deformations are found by applying the finite element method. One of the first models of this type was by Clausi and Brodland (1993), who modelled neurulation through an apical constriction mechanism. All the 
forces associated with the apical cytoskeleton were bundled into a force term active on the outer surface. By varying the parameters associated with this force the authors managed to reproduce various characteristic deformations in a relatively thick sheet of tissue. Davidson et al (1995) also used finite elements, in order to model invagination in a gastrulating sea urchin embryo. The purpose of the article was to distinguish between five various invagination mechanisms, and to determine which mechanisms were more effective in tissues of various stiffnesses. Apical constriction was one of these mechanisms and was modelled by imposing a change of shape on elements in the invaginating region.

Finally we note the recent contributions of Muñoz et al (2007) and Ramasubramanian and Taber (2008). In these models deforming embryos are modelled as a nonlinearly-elastic material using the finite element method. In both papers the deformation gradient is decomposed into a "growth" part and an "accommodation" part. The reason for this is that the growth may be an incompatible deformation which leaves the solid discontinuous; the accommodation restores the body's continuity. Such deformation gradient decomposition methods are widely used in biomechanical modelling; see Jones and Chapman (2009) for details. In both models, the growth phase need not refer to a physical growth, but rather to an active deformation that constricts the apical surface (and, in the work of Muñoz et al (2007), expands the basal surface and imposes an apical-basal elongation). Ramasubramanian and Taber propose that the "growth" term should be related to stress fields in the embryonic tissue through a feedback mechanism, where growth induces stresses which in turn either inhibit or promote growth.

In this article we will derive a thin elastic shell model for epithelia undergoing apical constriction. By explicitly accounting for the contractile fibres in the apical regions of the cells forming the epithelium, we hope to extend previous shell models in two ways. Firstly, the ad hoc surface forces that appear in other models (for example, see Zinemanas and Nir (1992)) would be replaced by a term derived directly from averaging the contribution of the tensions in each contractile fibre, and thus should be more physically realistic. Secondly, the fibres' contraction results in a higher density of fibres per unit (deformed) area in the epithelium, and thus alters the bending and stretching stiffnesses of the shell. Although the deformation indicated by the typical gastrulation process in Figure 1 is quite large, we will limit our analysis to linear strain-displacement relations. The equations derived here may be altered to include geometric nonlinearity, but the qualitative be- haviour of the tissue should not be altered by the assumption of linearity.

We will first, in Section 2, review the basic equations of shell theory. In the main section of this paper, Section 3, we will determine the effect of the embedded fibre-sheet on the overall behaviour of the shell, before analysing the stress-strain relationship arising due to the deformation of the fibres. We obtain new stretching and bending tensors, together with surface force terms which originate from the active contraction of the fibres, and in Section 4 simple numerical calculations are performed using this theory, in order to simulate representative tissue motions. Conclusions are drawn in Section 5 .

\section{An overview of shell theory}

As noted in the introduction, many of the investigations into epithelial mechanics have exploited the thinness of the tissue. If this approximation is applied to an elastic solid, the resulting theory is known as shell theory. In this section we will review the key results and terminology; a fuller account may be found in, for example, Niordson (1985).

In shell theory the displacement of the shell is given in terms of the displacement of its middle surface. An understanding of the geometry of surfaces is thus essential in analysing shell mechanics. A surface in threedimensional space can be defined by the position vector $\boldsymbol{r}=\boldsymbol{r}\left(x^{1}, x^{2}\right)$, where $x^{1}$ and $x^{2}$ are two coordinates that parametrise the surface. For example, a spherical surface of radius $R$ has the colatitudinal and azimuthal angles $\theta$ and $\phi$ respectively as its coordinates, and $\boldsymbol{r}=R \sin \theta\left(\cos \phi \boldsymbol{e}_{x}+\sin \phi \boldsymbol{e}_{y}\right)+R \cos \theta \boldsymbol{e}_{z}$ with respect to the Cartesian basis $\left(\boldsymbol{e}_{x}, \boldsymbol{e}_{y}, \boldsymbol{e}_{z}\right)$. The metric tensor of the surface, also known as the first fundamental form of the surface, is given by $a_{\alpha \beta}=\boldsymbol{r}_{, \alpha} \cdot \boldsymbol{r}_{, \beta}$, a subscript preceded by a comma denoting differentiation with respect to that particular coordinate. ${ }^{1}$ If we denote the unit normal to the surface by $\boldsymbol{n}$, the second fundamental form $d_{\alpha \beta}$ is defined by $d_{\alpha \beta}=\boldsymbol{n} \cdot \boldsymbol{r}_{, \alpha \beta}$. Using this tensor one may obtain ${ }^{2}$ the mean curvature $H=\frac{1}{2} d_{\alpha}^{\alpha}$ and the Gaussian curvature $K=\operatorname{det}\left(d_{\alpha \beta}\right) / \operatorname{det}\left(a_{\alpha \beta}\right)$.

The kinematics of the centre surface can be described by changes in the fundamental tensors $a_{\alpha \beta}$ and $d_{\alpha \beta}$. Suppose that quantities in the deformed state are distinguished by a superscript $*$. Then we define the

\footnotetext{
1 Greek indices range over 1,2 .

2 The summation convention holds throughout this article. Note that the raising or lowering of indices is achieved by multiplying with the metric tensor: $T_{\alpha}^{\gamma}=a^{\gamma \beta} T_{\beta \alpha}=a_{\alpha \beta} T^{\gamma \beta}$; the contravariant metric tensor $a^{\alpha \beta}$ is defined through $a^{\alpha \beta} a_{\beta \gamma}=\delta_{\gamma}^{\alpha}$, Kronecker's delta.
} 
strain tensor $\gamma_{\alpha \beta}=\frac{1}{2}\left(a_{\alpha \beta}^{*}-a_{\alpha \beta}\right)$ and the changeof-curvature tensor $\rho_{\alpha \beta}=\left(d_{\alpha \beta}^{*}-d_{\alpha \beta}\right)$. These can be written in terms of the components $\left(v_{\alpha}, w\right)$ of the displacement vector $\left(\boldsymbol{r}^{*}-\boldsymbol{r}\right)$ (where $w$ is the normal displacement and $v_{\alpha}$ are the in-surface displacement components referred to the base vectors $\left.\boldsymbol{a}^{\alpha}=a^{\alpha \beta} \boldsymbol{r}_{, \beta}\right)$. The full expressions are rather complicated (Niordson, 1985); keeping only linear terms we have

$\gamma_{\alpha \beta}=\frac{1}{2}\left(v_{\alpha \mid \beta}+v_{\beta \mid \alpha}\right)-d_{\alpha \beta} w$,

$\rho_{\alpha \beta}=w_{\mid \alpha \beta}+\left.d_{\alpha \gamma} v^{\gamma}\right|_{\beta}+\left.d_{\beta \gamma} v^{\gamma}\right|_{\alpha}+v^{\gamma} d_{\gamma \alpha \mid \beta}-d_{\beta \gamma} d_{\alpha}^{\gamma} w$.

(A subscript preceded by a vertical line represents covariant differentiation.) Which terms are kept in the expressions for $\gamma_{\alpha \beta}, \rho_{\alpha \beta}$ depends on the desired accuracy of the solution, ease of computation of results, and on the relative size of the terms.

Analysing the statics of the shell involves averaging the three-dimensional stress across the shell thickness and obtaining equivalent in-plane membrane stresses and moments which are applied to the shell centresurface. As per Niordson (1985), given a three-dimensional stress field $\sigma^{i j}$ in the shell, the membrane stress tensor $\mathcal{N}^{\alpha \beta}$ and the moment tensor $\mathcal{M}^{\alpha \beta}$ perform these roles, and are given by

$\mathcal{N}^{\alpha \beta}=\int_{-h / 2}^{h / 2} \sigma^{\alpha \gamma}\left(\delta_{\gamma}^{\beta}-d_{\gamma}^{* \beta} z\right)\left(1-2 H^{*} z+K^{*} z^{2}\right) \mathrm{d} z$,

$\mathcal{M}^{\alpha \beta}=-\int_{-h / 2}^{h / 2} z \sigma^{\alpha \gamma}\left(\delta_{\gamma}^{\beta}-d_{\gamma}^{* \beta} z\right)\left(1-2 H^{*} z+K^{*} z^{2}\right) \mathrm{d} z$

The equilibrium equations satisfied by the tensors (3)(4) are

$\mathcal{N}^{\alpha \beta}\left\|_{\alpha}+d^{* \beta}{ }_{\alpha} \mathcal{M}^{\gamma \alpha}\right\|_{\gamma}+F^{* \beta}=0$,

$\mathcal{M}^{\alpha \beta} \|_{\alpha \beta}-d_{\alpha \beta}^{*} \mathcal{N}^{\alpha \beta}-p^{*}=0$,

where $F^{\beta}$ is a vector of external forces acting tangentially to the shell, and $p$ is a pressure acting normally to the shell. The double line indicates covariant differentiation with respect to the deformed configuration.

The tensors $\mathcal{N}^{\alpha \beta}$ and $\mathcal{M}^{\alpha \beta}$ may be substituted into the expression arising from the principle of virtual work. A virtual displacement (infinitesimally small) gives rise to an infinitesimal strain tensor $\Delta \gamma_{\alpha \beta}$ and change-ofcurvature tensor $\Delta \rho_{\alpha \beta}$. The infinitesimal increase in energy caused by this deformation may be written

$\Delta V=\int_{\Omega}\left(N^{\alpha \beta} \Delta \gamma_{\alpha \beta}+M^{\alpha \beta} \Delta \rho_{\alpha \beta}\right) \mathrm{d} S$, where $\Omega$ is some region of the shell; this equation defines the effective membrane stress tensor $N^{\alpha \beta}$ and the effective moment tensor $M^{\alpha \beta}$. It can be shown that

$N^{\alpha \beta}=\mathcal{N}^{\alpha \beta}-d_{\gamma}^{* \beta} \mathcal{M}^{\gamma \alpha}, \quad M^{\alpha \beta}=\frac{1}{2}\left(\mathcal{M}^{\alpha \beta}+\mathcal{M}^{\beta \alpha}\right)$.

These satisfy the equilibrium conditions

$$
\begin{aligned}
& N^{\alpha \beta}\left\|_{\alpha}+2 d_{\gamma}^{* \beta} M^{\gamma \alpha}\right\|_{\alpha}+M^{\gamma \alpha} d_{\gamma}^{* \beta} \|_{\alpha}+F^{* \beta}=0, \\
& M^{\alpha \beta} \|_{\alpha \beta}-d_{\alpha \gamma}^{*} d_{\beta}^{* \gamma} M^{\alpha \beta}-d_{\alpha \beta}^{*} N^{\alpha \beta}-p^{*}=0 .
\end{aligned}
$$

Covariant differentiation with respect to the deformed shell can usually be replaced by that with respect to the undeformed shell, and we follow this practice here (although the distinction is important for elastic stability applications (Koiter, 1966)). The curvature tensor $d_{\alpha \beta}^{*}$ is equal to $d_{\alpha \beta}+\rho_{\alpha \beta}$. If we keep these terms in the equilibrium equations above, the equations will become nonlinear because the tensors $N^{\alpha \beta}$ and $M^{\alpha \beta}$ are (as we see shortly) proportional to the strain tensor and change of curvature tensor respectively. We will assume that the linear theory is sufficient to account for the shell's behaviour under apical constriction, and as such the equilibrium equations become

$$
\begin{aligned}
& \left.N^{\alpha \beta}\right|_{\alpha}+\left.2 d_{\gamma}^{\beta} M^{\gamma \alpha}\right|_{\alpha}+\left.M^{\gamma \alpha} d_{\gamma}^{\beta}\right|_{\alpha}+F^{\beta}=0, \\
& \left.M^{\alpha \beta}\right|_{\alpha \beta}-d_{\alpha \gamma} d_{\beta}^{\gamma} M^{\alpha \beta}-d_{\alpha \beta} N^{\alpha \beta}-p=0 .
\end{aligned}
$$

Now, as noted above the shell is assumed to behave according to the linear elastic theory, so that $W$, the strain energy density, is a quadratic function of $\gamma_{\alpha \beta}$ and $\rho_{\alpha \beta}$. Thus

$W=C^{\alpha \beta \lambda \mu} \gamma_{\alpha \beta} \gamma_{\lambda \mu}+D^{\alpha \beta \lambda \mu} \gamma_{\alpha \beta} \rho_{\lambda \mu}+F^{\alpha \beta \lambda \mu} \rho_{\alpha \beta} \rho_{\lambda \mu}$.

The fourth-order tensors in this expression are all functions of the shell geometry $\left(h, a_{\alpha \beta}, d_{\alpha \beta}\right)$ and its elastic constants. By comparing the relative sizes of each term, it can be shown that for an isotropic shell,

$$
\begin{aligned}
W \sim G h[( & \left.C_{1} a^{\alpha \beta} a^{\lambda \mu}+C_{2} a^{\alpha \lambda} a^{\beta \mu}\right) \gamma_{\alpha \beta} \gamma_{\lambda \mu} \\
& \left.+h^{2}\left(F_{1} a^{\alpha \beta} a^{\lambda \mu}+F_{2} a^{\alpha \lambda} a^{\beta \mu}\right) \rho_{\alpha \beta} \rho_{\lambda \mu}\right]
\end{aligned}
$$

with relative error at most $|h / R|+|h / \lambda|^{2}$, where $R$ is the shortest radius of curvature and $\lambda$ is the characteristic deformation wavelength; $G$ is the shear modulus of the material.

By comparison with the principle of virtual work, we have

$N^{\alpha \beta}=\frac{\partial W}{\partial \gamma_{\alpha \beta}}, \quad M^{\alpha \beta}=\frac{\partial W}{\partial \rho_{\alpha \beta}}$, 
SO

$N^{\alpha \beta}=2 G h\left(C_{1} a^{\alpha \beta} a^{\lambda \mu}+C_{2} a^{\alpha \lambda} a^{\beta \mu}\right) \gamma_{\lambda \mu} ;$

$M^{\alpha \beta}=2 G h^{3}\left(F_{1} a^{\alpha \beta} a^{\lambda \mu}+F_{2} a^{\alpha \lambda} a^{\beta \mu}\right) \rho_{\lambda \mu}$.

The constants $C_{i}$ and $F_{i}$ are found to be such that

$N^{\alpha \beta}=D A^{\alpha \beta \lambda \mu} \gamma_{\lambda \mu} ; \quad M^{\alpha \beta}=B A^{\alpha \beta \lambda \mu} \rho_{\lambda \mu}$,

where

$D=\frac{2 G h}{1-\nu}, \quad B=\frac{G h^{3}}{6(1-\nu)}$,

$A^{\alpha \beta \lambda \mu}=\left(\frac{1-\nu}{2}\right)\left(a^{\alpha \lambda} a^{\beta \mu}+a^{\alpha \mu} a^{\beta \lambda}\right)+\nu a^{\alpha \beta} a^{\lambda \mu}$,

and $G, \nu$ are respectively the shear modulus and Poisson ratio of the material.

In summary, the governing equations of shell theory are the equilibrium equations (11)-(12); the constitutive relations (18) that link stress and strain; and the strain-displacement relations (1)-(2). These are all to be solved simultaneously; in practice simplifications based on shell geometry are often made. For more complicated geometries a fully numerical implementation is essential.

\section{A modified shell theory to account for apical fibres}

Glancing at Figure 2, it is clear that treating the epithelial tissue as a homogeneous elastic material is rather simplistic. All the microscopic heterogeneities in the epithelial structure are lost. If those microscopic features are important for the mechanics, they must then be re-introduced in an ad hoc manner - for example, by modelling apical constriction through an abstract surface tension term. In this section we explicitly account for the fibres in the apical region of the cell to derive a more physically-realistic model for apical constriction.

We noted in the introduction that two mechanisms of apical constriction are specified in the literature: the contraction of a fine mesh of microfilaments distributed throughout the entire apical cortex; and the contraction of a circumferential bundle of fibres in each cell. These are shown in Figure 4. Strong attachments located in the junctional complex connect the apical cytoskeleton of neighbouring cells, and thus the cortical cytoskeleton in the entire epithelial tissue can be modelled as a continuous surface embedded in the tissue. The remaining tissue, formed from the cell cytoplasms, organelles, membranes and remaining cytoskeleton, will be assumed to behave as a linearly elastic solid. Again,
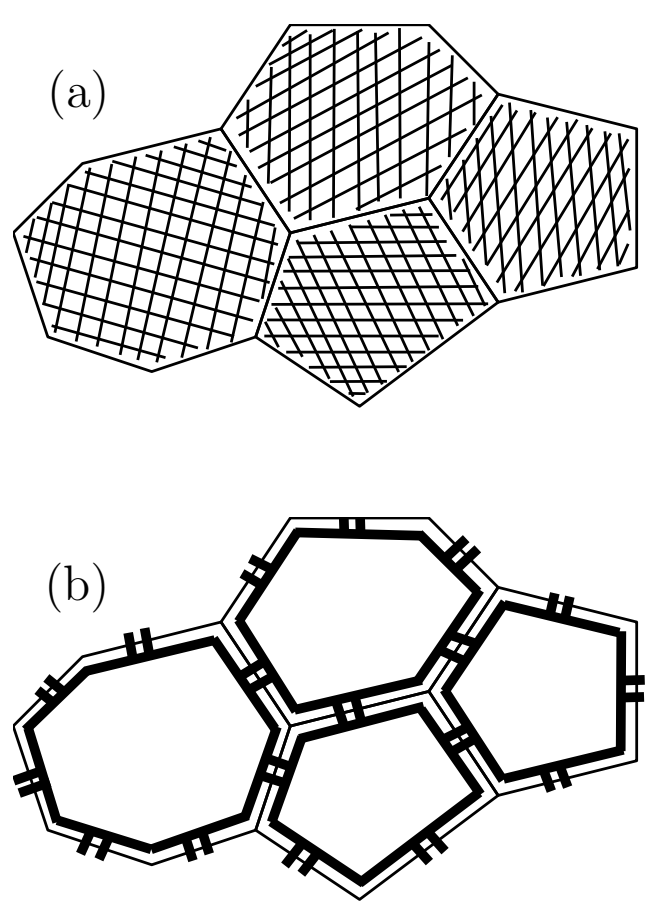

Figure 4 The same patch of cells (viewed from above the tissue) displaying the two canonical configurations for the apical fibres: (a) as a fine mesh of filaments, (b) as a circumferential bundle, connected to neighbouring cells by strong attachments.

this is rather simplistic but we do not believe that these components of the cell play an active role in apical constriction.

Thus the epithelial tissue will comprise a homogeneous linearly-elastic material of thickness $h$, with a surface embedded at a distance $\bar{z}$ from the centre-surface which deforms with the rest of the material, and can transmit force, in order to model the apical fibres. This simplified scenario is depicted in Figure 5.

To model such a tissue, we need to modify the expression for $\sigma^{\alpha \beta}$, the full three-dimensional stress tensor in the tissue. This is subsequently integrated through the tissue thickness, as in (3)-(4), to find the stress measures of the shell. which pertain only to the centresurface of the tissue. For a standard shell (as indicated in Section 2), $\sigma^{\alpha \beta}$ is linear in the three-dimensional strain tensor, according to the hypothesis of linear elasticity. In our model for epithelial tissue we denote this linear component by $\sigma_{\mathrm{b}}^{\alpha \beta}$, to account for the mechanical properties of the bulk of the tissue. However, we also need to consider the embedded fibre layer. Thus we add to $\sigma_{\mathrm{b}}^{\alpha \beta}$ a term $\Sigma^{\alpha \beta} \delta(z-\bar{z})$, a force per unit length (the two-dimensional stress equivalent) concentrated at the 


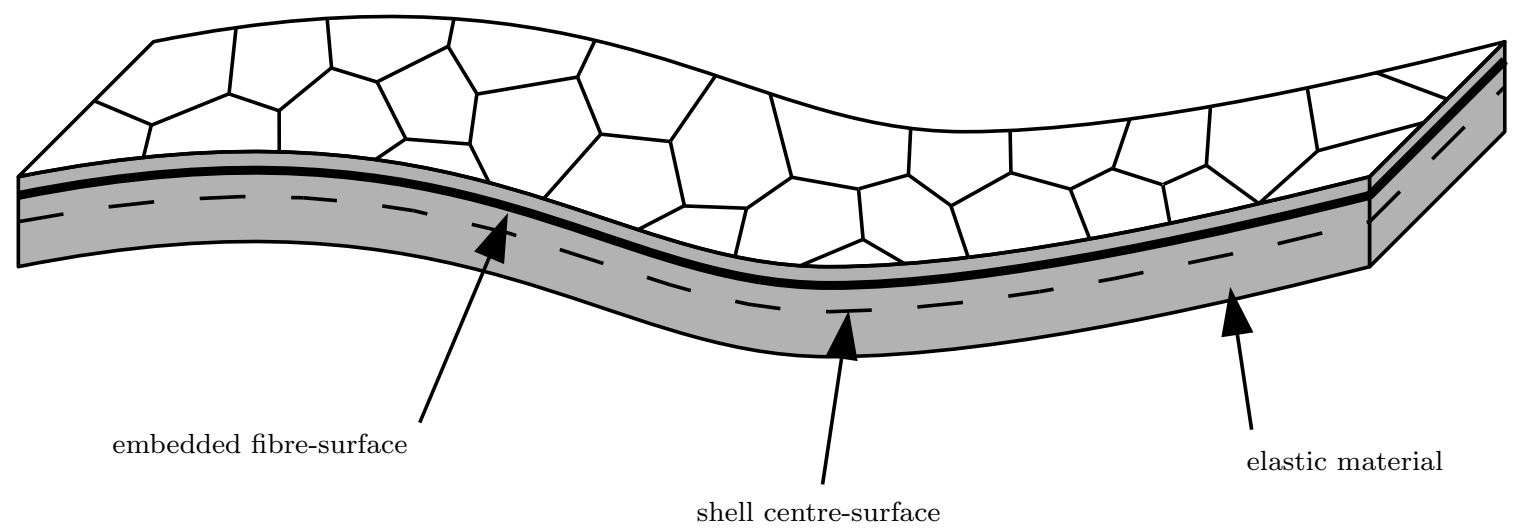

Figure 5 A shell model containing an embedded fibre membrane.

location of an embedded membrane. Overall, we have

$\sigma^{\alpha \beta}=\sigma_{\mathrm{b}}^{\alpha \beta}+\Sigma^{\alpha \beta} \delta(z-\bar{z})$,

$\sigma^{3 i}=\sigma^{i 3}=0$,

where $\delta(\cdot)$ is the Dirac delta function. The embedded layer should not produce any out-of-surface stress components.

Expressions (21)-(22) are then integrated through the thickness of the material to find our shell stress measures. From (3), (4) and (8) we obtain (by linearity) decompositions of $N^{\alpha \beta}$ and $M^{\alpha \beta}$ into terms which arise from the bulk of the tissue and from the embedded fibre layer:

$N^{\alpha \beta}=N_{\mathrm{b}}^{\alpha \beta}+N_{\Sigma}^{\alpha \beta}, \quad M^{\alpha \beta}=M_{\mathrm{b}}^{\alpha \beta}+M_{\Sigma}^{\alpha \beta}$,

where $N_{\mathrm{b}}^{\alpha \beta}, M_{\mathrm{b}}^{\alpha \beta}$ are the results of (3), (4) and (8) using $\sigma_{\mathrm{b}}^{\alpha \beta}$ rather than $\sigma^{\alpha \beta}$, and

$$
\begin{aligned}
N_{\Sigma}^{\alpha \beta}=\Sigma^{\gamma \delta}\left(\delta_{\delta}^{\lambda}-d_{\delta}^{* \lambda} \bar{z}\right)(1- & \left.2 H^{*} \bar{z}+K^{*} \bar{z}^{2}\right) \\
& \times\left(\delta_{\lambda}^{\beta} \delta_{\gamma}^{\alpha}+\delta_{\lambda}^{\alpha} d_{\gamma}^{* \beta} \bar{z}\right)
\end{aligned}
$$

$$
\begin{aligned}
M_{\Sigma}^{\alpha \beta}= & -\frac{\bar{z}}{2}\left(1-2 H^{*} \bar{z}+K^{*} \bar{z}^{2}\right) \\
& \times\left(\Sigma^{\alpha \gamma}\left(\delta_{\gamma}^{\beta}-d_{\gamma}^{* \beta} \bar{z}\right)+\Sigma^{\beta \gamma}\left(\delta_{\gamma}^{\alpha}-d_{\gamma}^{* \alpha} \bar{z}\right)\right) .
\end{aligned}
$$

Since the bulk of the shell is linearly elastic, we will henceforth write

$N_{\mathrm{b}}^{\alpha \beta}=D A^{\alpha \beta \lambda \mu} \gamma_{\lambda \mu}, \quad M_{\mathrm{b}}^{\alpha \beta}=B A^{\alpha \beta \lambda \mu} \rho_{\lambda \mu}$,

with $D$ and $B$ given by (19). We denote the bulk elastic constants by $G$ and $\nu$.

In general, $N_{\Sigma}^{\alpha \beta}$ and $M_{\Sigma}^{\alpha \beta}$ will be dependent on both $\gamma_{\alpha \beta}$ and $\rho_{\alpha \beta}$, since $d_{\alpha \beta}^{*}$ (and thus $H^{*}$ and $K^{*}$ ) is referred to the deformed configuration. Thus as well as providing terms which will appear as external forces in the equilibrium equations (11)-(12), (simulating the active bending and stretching of the tissue by the fibres), the constitutive relations (18) will also be modified (modelling the changed density of fibres after a contraction event).

\subsection{Modelling the embedded sheet}

In this section we will obtain an expression for $\Sigma^{\alpha \beta}$. In other words, if we have a membrane which is undergoing a certain deformation, we will determine the tension produced in the membrane, under the assumption that the membrane is composed of a collection of fibres which have the ability to contract.

Philosophically this is the same approach as considered by Lanir (1983) and by Brodland and Wiebe (2004). Lanir derived a mechanical model for a fibrous tissue, while Brodland and Wiebe considered a simple planar epithelial tissue. The cells in the latter model were polygonal and as such the tissue was considered to be a tessellation of these polygons; a constant force was presumed to act along the edges of each cell in the tessellation. As the tissue was deformed, the cells were also deformed, in a proportionate manner. The new effective stress in the tissue could could be calculated by taking a cross-section and calculating the sum of the forces acting on that surface.

We will perform a similar analysis, but with a few significant differences. Firstly, our focus will be a curved tissue, although this should not present any fundamental difficulties. Secondly, the forces in the network of fibres will not be assumed constant, as in Brodland and Wiebe, but will be assumed to be a certain function of their deformation, as done by Lanir. In contrast to 
Lanir, however, we will include fibre contraction and (explicitly) the length of the fibres.

The surface representing the embedded membrane of fibres will, as usual, have a reference configuration (with base vectors $\boldsymbol{g}_{i}$ ) and a deformed configuration (with base vectors $\boldsymbol{g}_{i}^{*}$ ), as shown in Figure 6 . Note that we have used a different notation $\left(\boldsymbol{g}_{i}\right)$ to the previous section $\left(\boldsymbol{a}_{i}\right)$ because the base vectors $\boldsymbol{a}_{i}$ refer to the middle-surface of the shell, not the embedded fibre layer, which is at a distance $\bar{z}$ from the middlesurface. The two descriptions are linked through $g_{\alpha \beta}=$ $a_{\alpha \beta}-2 d_{\alpha \beta} \bar{z}+d_{\alpha}^{\gamma} d_{\gamma \beta} \bar{z}^{2}$. We also introduce the strain tensor in the fibre-layer as

$$
\begin{aligned}
\bar{\gamma}_{\alpha \beta}= & \frac{1}{2}\left(g_{\alpha \beta}^{*}-g_{\alpha \beta}\right) \sim \gamma_{\alpha \beta}-\bar{z} \rho_{\alpha \beta} \\
& +\bar{z}^{2}\left[\frac{1}{2}\left(\rho_{\alpha}^{\gamma} d_{\gamma \beta}+\rho_{\beta}^{\gamma} d_{\gamma \alpha}\right)-\gamma^{\gamma \sigma} d_{\sigma \alpha} d_{\gamma \beta}\right] .
\end{aligned}
$$

The typical lengthscale of the tissue at the level depicted in Figure 6 will be denoted $L$. On the scale of the cells, the lengthscale will be $l$, the typical diameter of a cell. We assume that $l \ll L$ so that we may analyse the problem in the asymptotic limit $l / L \rightarrow 0$. Now, as noted in Figure 4, the microstructure of the embedded membrane is either a fine mat of cytoskeletal fibres or a tessellation of cells. Either way, the membrane is composed of a collection of force-carrying elements (henceforth "fibres") which represent the individual cytoskeletal fibres in the first case, and the cell edges in the tessellation case.

We represent a typical fibre by a vector $\boldsymbol{Q}$, embodying the length and orientation of the fibre. The corresponding vector in the deformed configuration is $\boldsymbol{Q}^{*}$, and henceforth all quantities in the deformed configuration will be distinguished by the addition of an asterisk. We assume that the number of fibres per unit undeformed (or deformed) area is $\rho$ (or $\rho^{*}$ ) and that the distribution of lengths and directions of the fibres in the reference configuration is governed by the probability density function $p(\boldsymbol{Q})$, with $Q=|\boldsymbol{Q}| \in\left(0, Q_{\max }\right)$.

If the vector $\boldsymbol{Q}$ may be written as $Q^{\alpha} \boldsymbol{g}_{\alpha}$ in the undeformed configuration, then its deformed state will be given by $\boldsymbol{Q}^{*}=Q^{\alpha} \boldsymbol{g}_{\alpha}^{*}$, as $Q^{\alpha}$ are the Lagrangian coordinates of the vector. Then, on considering the change in the squared length of $\boldsymbol{Q}$, we recover the strain tensor of the surface: $Q^{* 2}-Q^{2}=Q^{\alpha} Q^{\beta}\left(g_{\alpha \beta}^{*}-g_{\alpha \beta}\right)=2 \bar{\gamma}_{\alpha \beta} Q^{\alpha} Q^{\beta}$. The component of the surface strain tensor in the direction of $\boldsymbol{Q}$ is given by $\bar{\gamma}_{\alpha \beta} Q^{\alpha} Q^{\beta} /|\boldsymbol{Q}|^{2}$. This quantity represents the change in length of the fibre $\boldsymbol{Q}$.

In obtaining this strain, the reference state of the fibres was assumed to be the same as that of the surface. However, we may model the active contraction of the fibres by assuming that the fibres change their reference state, represented by the alternative metric tensor $\lambda_{\alpha \beta}$. This allows us to incorporate not only a lengthen$\mathrm{ing} /$ shortening of the fibres, but also some directionality in the contraction, where the amount of contraction is dependent on the directionality of the fibres. This directionality is important for invaginations which take the form of a groove, for instance in neurulation and Xenopus gastrulation. An isotropic contraction is represented by $\lambda_{\alpha \beta}=\lambda g_{\alpha \beta}$ for $0<\lambda<1$.

With this hypothesis, we get

$$
\begin{aligned}
Q^{* 2}-Q^{2}=Q^{\alpha} Q^{\beta}\left(g_{\alpha \beta}^{*}-\lambda_{\alpha \beta}\right) & \\
=Q^{\alpha} Q^{\beta}\left(g_{\alpha \beta}^{*}-g_{\alpha \beta}\right) & +Q^{\alpha} Q^{\beta}\left(g_{\alpha \beta}-\lambda_{\alpha \beta}\right) \\
& =2 Q^{\alpha} Q^{\beta}\left(\bar{\gamma}_{\alpha \beta}+\eta_{\alpha \beta}\right),
\end{aligned}
$$

where $\eta_{\alpha \beta}=\frac{1}{2}\left(g_{\alpha \beta}-\lambda_{\alpha \beta}\right)$ represents the "additional strain" induced by the active contraction in the fibre. We define $\widetilde{\gamma}_{\alpha \beta}=\bar{\gamma}_{\alpha \beta}+\eta_{\alpha \beta}$, then the component of the surface strain tensor in the direction of $\boldsymbol{Q}$ is $\widetilde{e}:=$ $\widetilde{\gamma}_{\alpha \beta} Q^{\alpha} Q^{\beta} /|\boldsymbol{Q}|^{2}$. The tension in the fibre, we assume, is a function of $\widetilde{e}$, and as such we write $T(\widetilde{e})$ for this tension. At this stage we make no restriction on the form of $T(\cdot) .^{3}$

Next we will "zoom out" from the fibre level and return to the scale of the tissue itself. We choose an arbitrary infinitesimal line element $\mathrm{d} s$ embedded in the deformed surface; this element has normal $\boldsymbol{n}^{*}$ (embedded in the surface). Consider any fibre $\boldsymbol{Q}^{*}$ in the surface. We identify the position of the fibre as the centre of the vector $\boldsymbol{Q}^{*}$. In the limit $\left|\boldsymbol{Q}^{*}\right| \ll \mathrm{d} s$, a fibre will cross $\mathrm{d} s$ if it lies in the parallelogram of length $\mathrm{d} s$ and height $\left|\boldsymbol{Q}^{*} \cdot \boldsymbol{n}^{*}\right|$ identified in Figure 7 . We assume that the number of fibres crossing $\mathrm{d} s$ is very large (valid in the limit $\left|Q^{*}\right| \ll \mathrm{d} s$ ), and as such the number of fibres crossing $\mathrm{d} s$ is given by

$$
\iint_{\Omega} \rho^{*}\left|\boldsymbol{Q}^{*} \cdot \boldsymbol{n}^{*}\right| \mathrm{d} s p(\boldsymbol{Q}) \mathrm{d}^{2} \boldsymbol{Q} .
$$

In this expression, $\rho^{*}$ is the number of fibres per unit deformed area, and $\Omega$ is the domain of the probability density function $p(\boldsymbol{Q})$ defined earlier.

Analogously to this calculation, we can determine the total force acting on the line element $\mathrm{d} s$. The tension in one fibre is denoted $T(\widetilde{e})$, and thus the force directed along the fibre is $\boldsymbol{T}^{*}=T(\widetilde{e}) \boldsymbol{Q}^{*} /\left|\boldsymbol{Q}^{*}\right|$. By summing up the forces in each fibre crossing $\mathrm{d} s$, we may determine the total force acting on $\mathrm{d} s$. However, $\left(\boldsymbol{Q}^{*}\right.$.

\footnotetext{
3 A constant tensor $\eta_{\alpha \beta}$ represents fibre lengthening or shortening in two principal directions, namely the eigenvectors of $\eta_{\alpha \beta}$. If the dependence of the change in fibre length on the fibre orientation in the tissue is more complicated than this, the tensor $\eta_{\alpha \beta}$ must be prescribed as a function of the fibre orientation.
} 


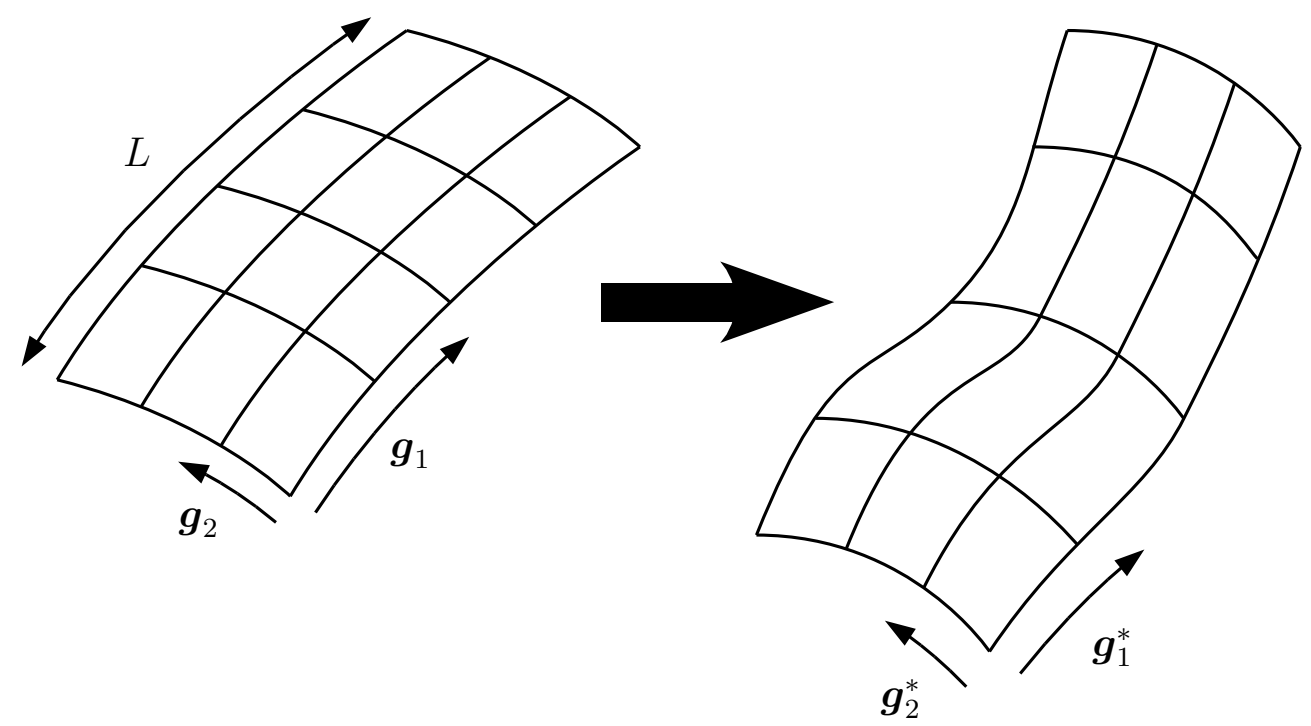

Figure 6 A depiction of part of the shell before and after deformation, displaying the base vectors.

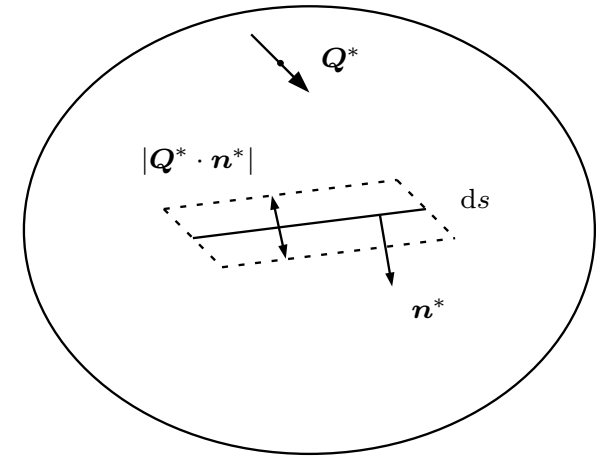

Figure 7 A representative volume element (RVE) of the surface, displaying the region in which $\boldsymbol{Q}^{*}$ (whose position is represented by a dot) must be positioned in order to cross $\mathrm{d} s$.

$\left.\boldsymbol{n}^{*}\right)$ may be positive or negative, and thus a similarlyoriented fibre may be counted as either a tensile or compressive force, whereas for (e.g.) $T(\widetilde{e})>0$ we would expect each element to count as a tensile force. To remove this contradiction we set

$\boldsymbol{T}^{*}=\frac{T(\widetilde{e}) \boldsymbol{Q}^{*}}{\left|\boldsymbol{Q}^{*}\right|}\left(\frac{\boldsymbol{Q}^{*} \cdot \boldsymbol{n}^{*}}{\left|\boldsymbol{Q}^{*} \cdot \boldsymbol{n}^{*}\right|}\right)$,

so that $\boldsymbol{T}^{*} \cdot \boldsymbol{n}^{*}$ has the same sign as $T(\widetilde{e})$. Utilising expression (29), the total force acting on $\mathrm{d} s$ is

$$
\begin{aligned}
\iint_{\Omega} \boldsymbol{T}^{*} \rho^{*}\left|\boldsymbol{Q}^{*} \cdot \boldsymbol{n}^{*}\right| \mathrm{d} s p(\boldsymbol{Q}) \mathrm{d}^{2} \boldsymbol{Q} \\
=\iint_{\Omega} \frac{T(\widetilde{e}) \boldsymbol{Q}^{*}}{\left|\boldsymbol{Q}^{*}\right|}\left(\boldsymbol{Q}^{*} \cdot \boldsymbol{n}^{*}\right) \rho^{*} \mathrm{~d} s p(\boldsymbol{Q}) \mathrm{d}^{2} \boldsymbol{Q} .
\end{aligned}
$$

Dividing this quantity by $\mathrm{d} s$ we obtain the force per unit length $\boldsymbol{t}^{*}$ acting on any line with (deformed) normal $\boldsymbol{n}^{*}$. Finally, using this $\boldsymbol{t}^{*}$, the in-surface term $\Sigma^{\alpha \beta}$ introduced earlier (the "two-dimensional stress") is identified from the relation $t^{* \alpha}=\Sigma^{\alpha \beta} n_{\beta}^{*}$, so that

$\Sigma^{\alpha \beta}=\iint_{\Omega} \frac{\rho^{*} T(\widetilde{e})}{\left|\boldsymbol{Q}^{*}\right|} Q^{\alpha} Q^{\beta} p\left(\boldsymbol{Q}^{*}\right) \mathrm{d}^{2} \boldsymbol{Q}$.

The area of an infinitesimal parallelogram of sides $\mathrm{d} u^{\alpha}$ before deformation is $\mathrm{d} A=g^{1 / 2} \mathrm{~d} u^{1} \mathrm{~d} u^{2}$ (where $\left.g=\operatorname{det}\left(g_{\alpha \beta}\right)\right)$, and post-deformation it becomes $\mathrm{d} A^{*}=$ $\left(g^{*}\right)^{1 / 2} \mathrm{~d} u^{1} \mathrm{~d} u^{2}$. Thus we can write the current density $\rho^{*}$ of fibres in terms of the pre-deformation density via $\rho^{*}=\left(g / g^{*}\right)^{1 / 2} \rho$, so that

$\Sigma^{\alpha \beta}=\rho\left(\frac{g}{g^{*}}\right)^{1 / 2} \iint_{\Omega} T(\widetilde{e}) \frac{Q^{\alpha} Q^{\beta}}{\left|\boldsymbol{Q}^{*}\right|} p(\boldsymbol{Q}) \mathrm{d}^{2} \boldsymbol{Q}$.

An additional manipulation can be made to this expression by writing $\boldsymbol{Q}=Q \boldsymbol{\Theta}$, where $Q=|\boldsymbol{Q}|$, or $Q^{\alpha}=$ $Q \Theta^{\alpha}$. In this formulation the quantity $\Theta^{\alpha}$ encompasses all information regarding the orientation of the fibres while the (pre-deformation) length is given by $Q$. Then, using $\boldsymbol{Q}^{*}=Q^{\alpha} \boldsymbol{g}_{\alpha}^{*}$, we obtain

$$
\Sigma^{\alpha \beta}=\rho \sqrt{\frac{g}{g^{*}}} \iint_{\Omega} Q T(\widetilde{e}) \frac{\Theta^{\alpha} \Theta^{\beta}}{\left(\Theta^{\kappa} \Theta^{\lambda} g_{\lambda \kappa}^{*}\right)^{1 / 2}} p\left(Q, \Theta^{\alpha}\right) \mathrm{d}^{2} \boldsymbol{Q} .
$$

We will simplify our calculations by assuming that the orientations of the fibres are uniformly distributed over 
$[0,2 \pi)$ in the undeformed configuration, and that $\Theta^{\alpha}$ are some functions of $\theta$, the angle with respect to some arbitrary axis in the undeformed configuration. Then $p\left(Q, \Theta^{\alpha}\right) \mathrm{d}^{2} \boldsymbol{Q}=\frac{1}{2 \pi} p(Q) \mathrm{d} Q \mathrm{~d} \theta$. Finally,

$\widetilde{e}=\widetilde{\gamma}_{\alpha \beta} Q^{\alpha} Q^{\beta} /|\boldsymbol{Q}|^{2}=\widetilde{\gamma}_{\alpha \beta} \Theta^{\alpha} \Theta^{\beta}$,

so $\widetilde{e}$ is independent of $Q$. Thus

$\Sigma^{\alpha \beta}=\frac{\rho}{2 \pi} \sqrt{\frac{g}{g^{*}}} \int_{0}^{2 \pi} \frac{T(\widetilde{e}) \Theta^{\alpha} \Theta^{\beta}}{\sqrt{\Theta^{\kappa} \Theta^{\lambda} g_{\kappa \lambda}^{*}}} \int_{0}^{Q_{\max }} Q p(Q) \mathrm{d} Q \mathrm{~d} \theta$

$=\frac{\rho \widehat{Q}}{2 \pi} \sqrt{\frac{g}{g^{*}}} \int_{0}^{2 \pi} \frac{T(\widetilde{e}) \Theta^{\alpha} \Theta^{\beta}}{\sqrt{\Theta^{\kappa} \Theta^{\lambda} g_{\kappa \lambda}^{*}}} \mathrm{~d} \theta$

where $\widehat{Q}$ is the mean fibre length in the reference configuration.

Our next step will be to assume that the strains are small. This will involve neglecting terms which are quadratic or higher in $\rho_{\alpha \beta}$ or $\gamma_{\alpha \beta}$. We assume that $T(0)=0$; this implies that

$T(\widetilde{e}) \sim k \widetilde{e}=k e+k \eta_{\mu \nu} \Theta^{\mu} \Theta^{\nu}$,

where $k=T^{\prime}(0)$ and $e=\Theta^{\kappa} \Theta^{\lambda} \bar{\gamma}_{\kappa \lambda}$. Additionally,

$\left(\Theta^{\kappa} \Theta^{\lambda} g_{\kappa \lambda}^{*}\right)^{-1 / 2}=(1+2 e)^{-1 / 2} \sim 1-e$

and $\left(g / g^{*}\right)^{1 / 2} \sim 1-\bar{\gamma}_{\kappa}^{\kappa}$. Then the integral in (37) simplifies in the small-strain limit so that

$$
\begin{aligned}
\Sigma^{\alpha \beta} \sim \frac{k \rho \widehat{Q}}{2 \pi}\left[\left(\eta_{\kappa \lambda}+\bar{\gamma}_{\kappa \lambda}-\eta_{\kappa \lambda} \bar{\gamma}_{\mu}^{\mu}\right) \overline{\mathcal{I}}^{\alpha \beta \kappa \lambda}\right. & \\
& \left.-\bar{\gamma}_{\kappa \lambda} \eta_{\mu \nu} \overline{\mathcal{I}}^{\alpha \beta \kappa \lambda \mu \nu}\right],
\end{aligned}
$$

where $\overline{\mathcal{I}}^{\alpha \beta \cdots \mu \nu}=\int_{0}^{2 \pi} \Theta^{\alpha} \Theta^{\beta} \cdots \Theta^{\mu} \Theta^{\nu} \mathrm{d} \theta$. To evaluate these integrals, we note that $\Theta=\Theta^{\alpha} \boldsymbol{g}_{\alpha}$. However, referred to a two-dimensional Cartesian basis $\boldsymbol{e}_{\alpha}$, we need $\boldsymbol{\Theta}=\Phi^{\alpha} \boldsymbol{e}_{\alpha}$, where $\Phi^{1}=\cos \theta, \Phi^{2}=\sin \theta$. The two bases are thus related through $\boldsymbol{e}_{\alpha}=m_{\alpha}^{\beta} \boldsymbol{g}_{\beta}$, where $m_{\alpha}^{\beta}=\boldsymbol{e}_{\alpha} \cdot \boldsymbol{g}^{\beta}$. Then we get $\Theta^{\alpha}=\Phi^{\gamma} m_{\gamma}^{\alpha}$, and consequently $\overline{\mathcal{I}}^{\alpha \beta \cdots \kappa \lambda}=m_{\mu}^{\alpha} m_{\nu}^{\beta} \cdots m_{\rho}^{\kappa} m_{\sigma}^{\lambda} \mathbb{I}^{\mu \nu \cdots \rho \sigma}$, where

$\mathbb{I}^{\mu \nu \cdots \rho \sigma}=\int_{0}^{2 \pi} \Phi^{\mu} \Phi^{\nu} \cdots \Phi^{\rho} \Phi^{\sigma} \mathrm{d} \theta$

The tensors $\mathbb{I}^{\mu \nu \cdots \rho \sigma}$ are isotropic Cartesian tensors of even rank, and as such (Suiker and Chang, 2000) are combinations of all possible products of Kronecker delta tensors. Furthermore, by symmetry the coefficients of the products are equal, and thus it can be shown that

$\mathbb{I}^{\kappa \lambda \mu \nu}=\frac{\pi}{4}\left(\delta_{\kappa \lambda} \delta_{\mu \nu}+\delta_{\kappa \mu} \delta_{\lambda \nu}+\delta_{\kappa \nu} \delta_{\lambda \mu}\right)$,

$$
\begin{aligned}
\mathbb{I}^{\kappa \lambda \mu \nu \rho \sigma}= & \frac{\pi}{24}\left[\delta_{\kappa \lambda}\left(\delta_{\mu \nu} \delta_{\rho \sigma}+\delta_{\mu \rho} \delta_{\nu \sigma}+\delta_{\mu \sigma} \delta_{\nu \rho}\right)\right. \\
& +\delta_{\kappa \mu}\left(\delta_{\lambda \nu} \delta_{\rho \sigma}+\delta_{\lambda \rho} \delta_{\nu \sigma}+\delta_{\lambda \sigma} \delta_{\nu \rho}\right) \\
& +\delta_{\kappa \nu}\left(\delta_{\lambda \mu} \delta_{\rho \sigma}+\delta_{\lambda \rho} \delta_{\mu \sigma}+\delta_{\lambda \sigma} \delta_{\mu \rho}\right) \\
& +\delta_{\kappa \rho}\left(\delta_{\lambda \mu} \delta_{\nu \sigma}+\delta_{\lambda \nu} \delta_{\mu \sigma}+\delta_{\lambda \sigma} \delta_{\mu \nu}\right) \\
& \left.\quad+\delta_{\kappa \sigma}\left(\delta_{\lambda \mu} \delta_{\nu \rho}+\delta_{\lambda \nu} \delta_{\mu \rho}+\delta_{\lambda \rho} \delta_{\mu \nu}\right)\right] .
\end{aligned}
$$

Then, using the fact that

$\delta_{\kappa \lambda} m_{\kappa}^{\alpha} m_{\lambda}^{\beta}=\left(\boldsymbol{e}_{\kappa} \cdot \boldsymbol{g}^{\alpha}\right)\left(\boldsymbol{e}_{\kappa} \cdot \boldsymbol{g}^{\beta}\right)=g^{\alpha \beta}$,

we find that $\overline{\mathcal{I}}^{\kappa \lambda \mu \nu}, \overline{\mathcal{I}}^{\kappa \lambda \mu \nu \rho \sigma}$ are as (42)-(43) but with $\delta_{\varepsilon \zeta}$ replaced by $g^{\varepsilon \zeta}$.

Thus we find that $\Sigma^{\alpha \beta}$ can be written as $\Sigma^{\alpha \beta}=$ $\Sigma_{0}^{\alpha \beta}+\mathcal{E}^{\alpha \beta \gamma \delta} \bar{\gamma}_{\gamma \delta}$, where

$\Sigma_{0}^{\alpha \beta}=\frac{k \rho \widehat{Q}}{2 \pi} \overline{\mathcal{I}}^{\alpha \beta \gamma \delta} \eta_{\gamma \delta}$

$\mathcal{E}^{\alpha \beta \gamma \delta}=\frac{k \rho \widehat{Q}}{2 \pi}\left[\overline{\mathcal{I}}^{\alpha \beta \gamma \delta}-\overline{\mathcal{I}}^{\alpha \beta \gamma \delta \mu \nu} \eta_{\mu \nu}-g^{\gamma \delta} \eta_{\mu \nu} \overline{\mathcal{I}}^{\alpha \beta \mu \nu}\right]$.

This expression for $\Sigma^{\alpha \beta}$ can now be substituted into (24)-(25) in order to find the additional terms in $N^{\alpha \beta}$ and $M^{\alpha \beta}$ that arise due to the presence of the fibre layer. The fibre-surface strain tensor $\bar{\gamma}_{\alpha \beta}$ may be expressed in terms of the centre-surface deformation tensors $\gamma_{\alpha \beta}, \rho_{\alpha \beta}$ through equation (27). The terms with an asterisk in (24)-(25) give rise to further terms in $\gamma_{\alpha \beta}$ and $\rho_{\alpha \beta}$, and the resulting formulae for $N^{\alpha \beta}$ and $M^{\alpha \beta}$ are further linearised with respect to these strain measures. The result is rather unwieldy and as such we do not record it here. However, we do notice that this particular result is far too comprehensive for our purposes. In particular, in obtaining the equivalent tensors for the isotropic shell case (see Section 2), certain terms were omitted as being small in comparison with $|h / R|+|h / \lambda|^{2}$. Thus, we should neglect the corresponding terms arising from the embedded fibre-surface: these terms would be the same size as the terms we have already omitted and so neglecting them wouldn't increase the relative error in our approximation. The result, after extensive algebraic manipulations, is

$N^{\alpha \beta}=N_{0}^{\alpha \beta}+\mathcal{D}^{\alpha \beta \lambda \mu} \gamma_{\lambda \mu}$,
$M^{\alpha \beta}=M_{0}^{\alpha \beta}+\mathcal{B}^{\alpha \beta \lambda \mu} \rho_{\lambda \mu}$,

where

$N_{0}^{\alpha \beta}=\Sigma_{0}^{\gamma \delta}\left(\delta_{\delta}^{\lambda}-d_{\delta}^{\lambda} \bar{z}\right)\left(1-2 H \bar{z}+K \bar{z}^{2}\right)\left(\delta_{\lambda}^{\beta} \delta_{\gamma}^{\alpha}+\delta_{\lambda}^{\alpha} d_{\gamma}^{\beta} \bar{z}\right)$,

$$
\begin{aligned}
M_{0}^{\alpha \beta}=- & \frac{\bar{z}}{2}\left(1-2 H \bar{z}+K \bar{z}^{2}\right) \\
& \times\left(\Sigma_{0}^{\alpha \gamma}\left(\delta_{\gamma}^{\beta}-\bar{z} d_{\gamma}^{\beta}\right)+\Sigma_{0}^{\beta \gamma}\left(\delta_{\gamma}^{\alpha}-\bar{z} d_{\gamma}^{\alpha}\right)\right),
\end{aligned}
$$




$$
\begin{array}{r}
\mathcal{D}^{\alpha \beta \lambda \mu}=D A^{\alpha \beta \lambda \mu}+\frac{k \rho \widehat{Q}}{2 \pi}\left(\mathcal{I}^{\alpha \beta \lambda \mu}-\eta_{\rho \sigma} \mathcal{I}^{\alpha \beta \lambda \mu \rho \sigma}\right. \\
\left.-\eta_{\rho \sigma} \mathcal{I}^{\alpha \beta \rho \sigma} a^{\lambda \mu}\right),
\end{array}
$$

$$
\begin{aligned}
\mathcal{B}^{\alpha \beta \lambda \mu}=B A^{\alpha \beta \lambda \mu} & +\frac{\bar{z}^{2} k \rho \widehat{Q}}{4 \pi}\left(2 \mathcal{I}^{\alpha \beta \lambda \mu}+a^{\beta \mu} \mathcal{I}^{\alpha \lambda \rho \sigma} \eta_{\rho \sigma}\right. \\
& \left.+a^{\alpha \mu} \mathcal{I}^{\beta \lambda \rho \sigma} \eta_{\rho \sigma}-2 \mathcal{I}^{\alpha \beta \lambda \mu \rho \sigma} \eta_{\rho \sigma}\right),
\end{aligned}
$$

and the terms $\mathcal{I}$ differ from $\overline{\mathcal{I}}$ in that they are formed from the middle-surface metric tensors $a^{\alpha \beta}$, not the fibre-surface tensors $g^{\alpha \beta}$.

The formulae (47)-(48) can now be substituted into the equilibrium equations (11)-(12). As a result we end up with new equilibrium equations where the active constriction terms $N_{0}^{\alpha \beta}, M_{0}^{\alpha \beta}$ are incorporated into the surface traction terms. Let $\tilde{N}^{\alpha \beta}=\mathcal{D}^{\alpha \beta \lambda \mu} \gamma_{\lambda \mu}, \widetilde{M}^{\alpha \beta}=$ $\mathcal{B}^{\alpha \beta \lambda \mu} \rho_{\lambda \mu}$, then we obtain

$\left.\widetilde{N}^{\alpha \beta}\right|_{\alpha}+\left.2 d_{\gamma}^{\beta} \widetilde{M}^{\gamma \alpha}\right|_{\alpha}+\left.\widetilde{M}^{\gamma \alpha} d_{\gamma}^{\beta}\right|_{\alpha}+\widetilde{F}^{\beta}=0$,

$\left.\widetilde{M}^{\alpha \beta}\right|_{\alpha \beta}-d_{\alpha \gamma} d_{\beta}^{\gamma} \widetilde{M}^{\alpha \beta}-d_{\alpha \beta} \widetilde{N}^{\alpha \beta}-\widetilde{p}=0$,

where

$\widetilde{F}^{\beta}=F^{\beta}+\left.N_{0}^{\alpha \beta}\right|_{\alpha}+\left.2 d_{\gamma}^{\beta} M_{0}^{\gamma \alpha}\right|_{\alpha}+\left.M_{0}^{\gamma \alpha} d_{\gamma}^{\beta}\right|_{\alpha}$,

$\widetilde{p}=p-\left.M_{0}^{\alpha \beta}\right|_{\alpha \beta}+d_{\alpha \gamma} d_{\beta}^{\gamma} M_{0}^{\alpha \beta}+d_{\alpha \beta} N_{0}^{\alpha \beta}$.

In these expressions, $F^{\beta}, p$ represent the applied tractions, while the remaining terms are fictitious tractions that model the apical constriction. We can thus represent the apical constriction process using standard shell theory, with modified constitutive tensors $\mathcal{D}^{\alpha \beta \lambda \mu}$, $\mathcal{B}^{\alpha \beta \lambda \mu}$, and a surface traction represented by $\widetilde{F}^{\beta}, \widetilde{p}$. The constitutive tensors account for the changing density of fibres during constriction while the force generated by the contracting fibres appear in the modified surface tractions. In Section 4 we will apply this modified shell theory to certain canonical morphogenetic processes, but first we will write down the equations for the simplest shell of all — a flat plate.

\subsection{Simplification for a plate}

In a plate, we have zero curvature, so that $d_{\alpha \beta}=0$. Furthermore, we use Cartesian coordinates, so that $a_{\alpha \beta}=$ $g_{\alpha \beta}=\delta_{\alpha \beta}$, the Kronecker delta. Under these assumptions, equations (49)-(56) become

$\left.\widetilde{N}^{\alpha \beta}\right|_{\alpha}+\widetilde{F}^{\beta}=0$ $\left.\widetilde{M}^{\alpha \beta}\right|_{\alpha \beta}-\widetilde{p}=0$

$\widetilde{F}^{\beta}=F^{\beta}+\left.N_{0}^{\alpha \beta}\right|_{\alpha}$, $\widetilde{p}=p-\left.M_{0}^{\alpha \beta}\right|_{\alpha \beta}$

$N_{0}^{\alpha \beta}=\Sigma_{0}^{\alpha \beta}, \quad M_{0}^{\alpha \beta}=-\bar{z} \Sigma_{0}^{\alpha \beta}, \quad \Sigma_{0}^{\alpha \beta}=\frac{k \rho \widehat{Q}}{2 \pi} \mathbb{I}^{\alpha \beta \gamma \delta} \eta_{\gamma \delta}$, and (51) and (52) remain the same (with $\mathcal{I}_{0}^{\alpha \cdots \eta}=\mathbb{I}^{\alpha \cdots \eta}$ ).

We will now show how this theory may be placed in the formulation of growth described by Dervaux et al (2009). They considered a plate which was growing, as described by a multiplicative decomposition of the deformation gradient $\left(\boldsymbol{F}=\boldsymbol{F}_{\mathrm{e}} \boldsymbol{F}_{\mathrm{g}}\right)$, briefly mentioned in Section 1. Under certain assumptions regarding the thinness of the material, they derived a version of the Föppl-von Kármán (FvK) equations:

$B\left(\nabla^{4} w-\nabla^{2} C_{\mathrm{M}}\right)-2 h[\chi, w]=p$,

$\nabla^{4} \chi+E\left([w, w]-C_{\mathrm{G}}\right)=0$.

Here, $\chi$ is an Airy stress function;

$$
\begin{aligned}
{[X, Y]=} & \left.\left.\frac{1}{2} \varepsilon^{\alpha \lambda} \varepsilon^{\beta \mu} X\right|_{\alpha \beta} Y\right|_{\lambda \mu} \\
& =\frac{1}{2}\left(X_{, 11} Y_{, 22}-2 X_{, 12} Y_{, 12}+X_{, 22} Y_{, 11}\right)
\end{aligned}
$$

$\varepsilon^{\alpha \beta}$ is the two-dimensional alternating tensor; $C_{\mathrm{M}}$ and $C_{\mathrm{G}}$ are growth terms arising from the growth tensor $\boldsymbol{F}_{\mathrm{g}} ; E=2 G(1+\nu)$ is the three-dimensional Young's modulus; and $B, w, p$ are as in our shell theory derived above. Note that these equations are nonlinear. In order to compare our (linear) theory to this modified FvK theory, we must add nonlinear terms to our equations. Specifically, we require that (1) be replaced by $\gamma_{\alpha \beta}=\frac{1}{2}\left(v_{\alpha \mid \beta}+v_{\beta \mid \alpha}\right)-d_{\alpha \beta} w+\frac{1}{2} w_{, \alpha} w_{, \beta}$, and (12) by $\left.M^{\alpha \beta}\right|_{\alpha \beta}-\left(d_{\alpha \beta}+\left.w\right|_{\alpha \beta}\right) N^{\alpha \beta}-p=0$. These equations now describe the classical shallow shell theory (Koiter, 1966). Furthermore, we ignore the effect that the embedded apical surface has on $\mathcal{D}^{\alpha \beta \lambda \mu}$ and $\mathcal{B}^{\alpha \beta \lambda \mu}$, keeping only the first terms in (51) and (52). Under these assumptions (and the omission of tangential surface loads), the plate equations become

$B \nabla^{4} w-2 h[w, \chi]-p+\left.M_{0}^{\alpha \beta}\right|_{\alpha \beta}=0$,

$\nabla^{4} \chi+E[w, w]+\left.E \varepsilon^{\alpha \beta} \varepsilon^{\gamma \mu} H_{\alpha \mu \rho \sigma} N_{0}^{\rho \sigma}\right|_{\beta \gamma}=0$,

where $\chi$ is such that $N^{\alpha \beta}=\left.h \varepsilon^{\alpha \lambda} \varepsilon^{\beta \mu} \chi\right|_{\lambda \mu}$, and $H_{\alpha \beta \gamma \delta}$ is the compliance tensor for stretching $\left(\gamma_{\alpha \beta}=H_{\alpha \beta \gamma \delta} \tilde{N}^{\gamma \delta}\right)$. Comparing this result with (60)-(61), we have

$\nabla^{2} C_{\mathrm{M}}=\frac{\bar{z} k \rho \widehat{Q}}{8 B}\left(\left.\eta_{\beta}^{\beta}\right|_{\alpha} ^{\alpha}+\left.2 \eta^{\alpha \beta}\right|_{\alpha \beta}\right)$,

$C_{\mathrm{G}}=-\frac{k \rho \widehat{Q}}{8 E h}\left[2(1+\nu) \varepsilon^{\kappa \beta} \varepsilon^{\gamma \lambda}-(1-3 \nu) a^{\beta \gamma} a^{\kappa \lambda}\right] \eta_{\kappa \lambda \mid \beta \gamma}$.

Under the further assumption that the constriction is isotropic $\left(\eta_{\alpha \beta}=\eta g_{\alpha \beta}\right)$,

$C_{\mathrm{M}}=\frac{\bar{z} k \rho \widehat{Q}}{2 B} \eta, \quad C_{\mathrm{G}}=\frac{k \rho \widehat{Q}(1-\nu)}{2 E h} \nabla^{2} \eta$. 

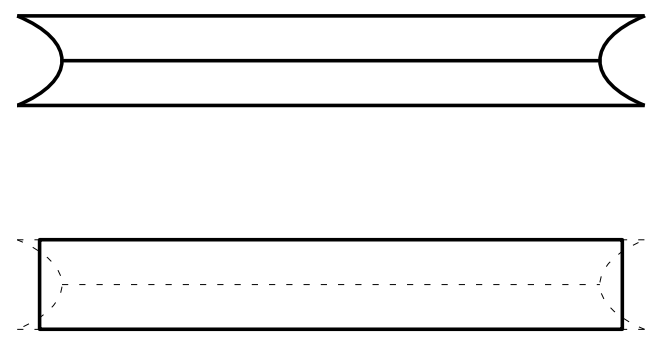

Figure 8 An edge-on diagram displaying how a plate with a constricting fibre-surface at its centre (top) is equivalent to a contracting plate (bottom).

We have thus shown that the apical constriction process is equivalent to growth as described by a certain deformation gradient decomposition. In particular, one may describe a growth mechanism by prescribing a certain $\eta_{\alpha \beta}$. For instance, by setting $\bar{z}=0$ (imagining a fibre-surface embedded at the centre-surface of the shell), one may describe an in-surface growth (or contraction) of the shell by introducing an appropriate $\eta_{\alpha \beta}$. A simple diagram (Figure 8) shows why this is physically realistic: as the embedded surface contracts, it drags the plate with it. But the shell equations are averaged through the shell thickness and thus on the scale of the shell only an in-surface contraction is observed. ${ }^{4}$

\subsubsection{Using a plate model to determine mechanical properties}

In numerical calculations of apical constriction, values for the mechanical parameters $G, \nu, k, \rho$ and $\widehat{Q}$ will of course be required, together with the properties of the surrounding medium. Such values are notably hard to obtain, given the inherent fragility of embryonic tissue. Of the few papers to investigate mechanical properties epithelial tissue, Wiebe and Brodland (2005) subjected amphibian tissue samples to uniaxial stretching and measured the response. In another investigation Davidson et al (1999) measured the elastic modulus of both the cell layer and the extracellular matrix of a sea urchin embryo through a compressive test. Perhaps understandably, the least-well understood components are the individual fibres in the apical region (whose properties we have subsumed into the parameter $k \rho \widehat{Q}$ ); one of the few studies into this aspect of cell mechanics is Burnside (1971). Experimentally-determined values for

\footnotetext{
4 The location of the surface at $\bar{z}=0$ means that the contraction induces no bending moments.
}

the elastic moduli of an isolated cell vary wildly; see Lim et al (2006) for an overview.

In light of the paucity of data on the mechanical properties of epithelia, it is thus inevitable that we must make assumptions on the values of the material constants in the model of Section 3. We will follow the principle that the larger the sample, the more reliable the data, and thus we disregard the data on the properties of the apical cytoskeleton. These properties will thus have to be deduced from other sources, in the following way. Wiebe and Brodland, as noted above, calculated the response of an embryonic epithelial sheet to uniaxial deformation, and thus calculated the twodimensional Young's modulus $\widetilde{E}$, which was found to be in the range $\left(1 \times 10^{-3}-8 \times 10^{-3}\right) \mathrm{N} \mathrm{m}^{-1}$. This response encapsulates the (linear) elastic behaviour both of the bulk of the cells and the apical layer, which we have treated as separate.

This experiment can be modelled analytically using the equations derived in Section 3.1 applied to a plate, with $\eta_{\alpha \beta}=0$ (as no apical constriction occurs during the experiment) and $\gamma_{\alpha \beta}=$ constant (as the uniaxial deformation is homogeneous). The two-dimensional Young's modulus can be found as a function of the elastic constants $G, \nu, k, \rho, \widehat{Q}$ of the epithelial sheet:

$$
\begin{aligned}
\widetilde{E}=\left[\frac{2 G h}{(1-\nu)}+\frac{3 k \rho \widehat{Q}}{8}\right]^{-1} & \left(4 G h+\frac{k \rho \widehat{Q}}{2}\right) \\
& \times\left[\frac{G h(1+\nu)}{(1-\nu)}+\frac{k \rho \widehat{Q}}{4}\right] .
\end{aligned}
$$

Now, the stiffness $G$ of cells, as collated by Lim et al (2006) varies from around $10 \mathrm{~Pa}$ to around $500 \mathrm{~Pa}$. We will take a value in this range as representative, and similarly for $\widetilde{E}$. The Poisson ratio $\nu$ is chosen to be 0.49 to model the near-incompressibility of the cells, and the thickness $h$ is chosen from the geometry of the problem. Equation (69) can then be solved to find a representative value for the apical fibre layer mechanical parameter $k \rho \widehat{Q}$. We need not consider the components of $k \rho \widehat{Q}$ separately as they always appear as a product. We note that if $2 G h(1+\nu) \geqslant \widetilde{E}$, we obtain $k \rho \widehat{Q} \leqslant$ 0 , which is clearly unphysical. Therefore care must be taken in choosing representative values of $G$ and $\widetilde{E}$ for a given value of the tissue thickness $h$.

\section{Numerical calculations}

We will now implement the results of the previous section in a numerical method. Applying the equations in their most general form yields a system that is overly 
complicated, and thus we will make a number of simplifications that make the calculations rather more simple. Firstly, linear shell theory is assumed, so that $\gamma_{\alpha \beta}$ and $\rho_{\alpha \beta}$ are purely linear functions of the shell displacements, given by (1) and (2). We also limit our consideration to simple geometries, namely a plate, and cylindrical and spherical shells. Furthermore, the problem is assumed to be invariant in one direction, thus simplifying the partial differential equation system into a system of ordinary differential equations (ODEs).

\subsection{Bending of a plate}

For simulation of plate bending by the mechanism of apical constriction, we simplify the system of equations to two ODEs by assuming all quantities are independent of $y$, the second Cartesian direction in the plane of the plate. If the constriction tensor is prescribed to be $\eta_{\alpha \beta}=f(x) \delta_{1 \alpha} \delta_{1 \beta}$, modelling a constriction of $x$ directed fibres but not of $y$-directed fibres (with the constriction of intermediately-directed fibres greater if they are more $x$-aligned), then the equations for the out-ofplane displacement $W(x)$ and the $x$-displacement $V(x)$ become

$$
\begin{aligned}
& {\left[\frac{2 G h}{1-\nu}+\frac{k \rho \widehat{Q}}{16}(6-11 f(x))\right] V^{\prime \prime}(x)} \\
& -\frac{11 k \rho \widehat{Q}}{16} f^{\prime}(x) V^{\prime}(x)+\frac{3 k \rho \widehat{Q}}{8} f^{\prime}(x)+F^{1}(x)=0, \\
& {\left[\frac{G h^{3}}{6(1-\nu)}+\frac{\bar{z}^{2} k \rho \widehat{Q}}{16}(6+f(x))\right] W^{\prime \prime \prime \prime}(x)} \\
& +\frac{\bar{z}^{2} k \rho \widehat{Q}}{8} f^{\prime}(x) W^{\prime \prime \prime}(x)+\frac{\bar{z}^{2} k \rho \widehat{Q}}{16} f^{\prime \prime}(x) W^{\prime \prime}(x) \\
& -\frac{3 \bar{z} k \rho \widehat{Q}}{8} f^{\prime \prime}(x)=p(x) .
\end{aligned}
$$

For numerical calculations, the tangential force $F^{1}(x)$ is assumed to be zero, and the normal traction $p(x)$ is set to $-\kappa W(x)$ : a Winkler foundation model to represent an elastic substrate. The equations may then be nondimensionalised using the scalings $(W, V)=L(\widehat{W}, \widehat{V})$, $x=L \widehat{x}, \bar{z}=h \widehat{\bar{z}}$, where $L$ is a characteristic length scale, yielding ${ }^{5}$

$$
\begin{gathered}
{\left[\frac{1}{6}+\frac{\alpha \widehat{\bar{z}}^{2}}{16}(6+f(\widehat{x}))\right] \widehat{W}^{\prime \prime \prime \prime}(\widehat{x})+\frac{\alpha \widehat{\bar{z}}^{2}}{8} f^{\prime}(\widehat{x}) \widehat{W}^{\prime \prime \prime}(\widehat{x})} \\
+\frac{\alpha \widehat{\bar{z}}^{2}}{16} f^{\prime \prime}(\widehat{x}) \widehat{W}^{\prime \prime}(\widehat{x})-\frac{3 \alpha \widehat{\bar{z}}}{8 \varepsilon} f^{\prime \prime}(\widehat{x})+\beta \widehat{W}(\widehat{x})=0,
\end{gathered}
$$

\footnotetext{
5 The constriction function $f(x)$ is already dimensionless.
}
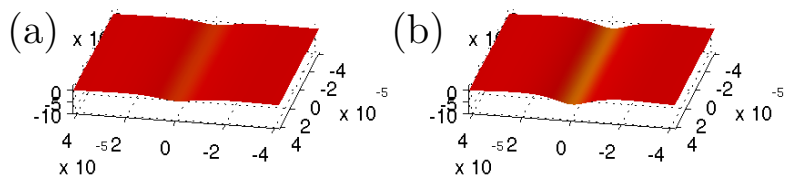

(c)

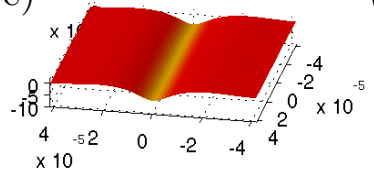

(d)

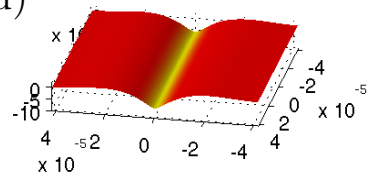

Figure 9 Increasing plate bending due to apical constriction. The surfaces (a)-(d) represent $\widetilde{f}$ increasing through $0.15,0.3$, $0.45,0.6$ respectively. The surface is colour-coded according to the value of $f(x)$ at that point; lighter colours represent more constriction.

$$
\begin{array}{r}
{\left[2+\frac{\alpha}{16}(6-11 f(\widehat{x}))\right] \widehat{V}^{\prime \prime}(\widehat{x})-\frac{11 \alpha}{16} f^{\prime}(\widehat{x}) \widehat{V}^{\prime}(\widehat{x})} \\
+\frac{3 \alpha}{8} f^{\prime}(\widehat{x})=0
\end{array}
$$

where

$\alpha=\frac{k \rho \widehat{Q}(1-\nu)}{G h}, \quad \varepsilon=\frac{h}{L}, \quad \beta=\frac{L^{4} \kappa(1-\nu)}{G h^{3}}$.

We choose values of $\alpha$ and $\beta$ consistent with the principles of Section 3.2.1, and choose $L$ to be the dimensions of a typical embryo, i.e. $L \approx 4 \times 10^{-5} \mathrm{~m}$. The plate is given a length of $2 L$ in the $x$-direction, and the constriction function $f(x)$ is prescribed to be $f(x)=$ $\widetilde{f} \exp \left(x^{2} /\left(a^{2} x^{2}-a^{4}\right)\right)$ in the region $|x|<a$, and zero for $|x| \geqslant a$ (where $a=0.4 L$ for computational purposes). This profile models an infinitely-differentiable function which is zero at $x= \pm a$ and increases to a maximum of $\widetilde{f}$ at $x=0$.

We solve (72) and (73) as a boundary value problem (applying clamped conditions at the edges) using the pre-existing boundary-value problem solution suite in the numerical computation software package Matlab. Figure 9 displays the quasistatic bending of a plate by the gradual increase of $\widetilde{f}$, indicating that as the degree of constriction in the apical layer increases, so does the amount of bending in the whole shell.

\subsection{Apical constriction in a cylindrical shell}

Another physically-relevant situation is that of bending in a cylindrical shell. The shell coordinates are $\theta$ (azimuth) and $z$ respectively, and all quantities will be 
(a)
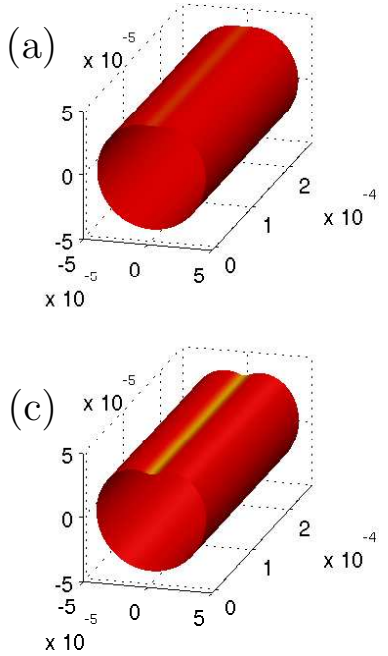

(b)

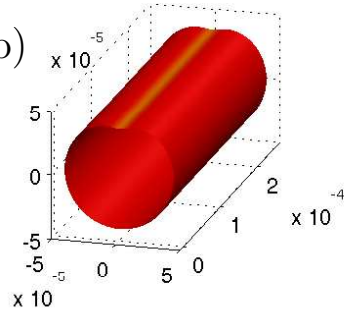

(d)

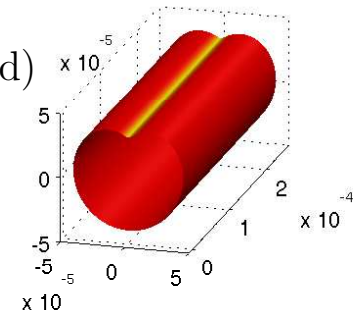

Figure 10 Increasing bending in a cylindrical shell due to apical constriction. The surfaces (a)-(d) represent $\widetilde{f}$ increasing through $0.15,0.3,0.45,0.6$ respectively. Colour is related to $f(x)$ as for Figure 9.

declared independent of $z$. Similarly to the plate, we prescribe the constriction to be $\eta_{\alpha \beta}=L^{2} f(\theta) \delta_{1 \alpha} \delta_{1 \beta}$, so that $z$-directed fibres do not contract. The radius of the cylinder is $L$, and appears in the definition of $\eta_{\alpha \beta}$ since $\boldsymbol{g}_{\alpha}$ are not unit vectors. A Winkler foundation is applied, and the resulting system of ODEs is nondimensionalised. The resulting equations are not related here for reasons of space, but the numerical results from Matlab (for the same form of $f(\theta)$ as for the plate) are shown in Figure 10. This type of deformation is reminiscent of ventral furrow formation in Drosophila. It may be instructive to perform numerical experiments with different constriction patterns, to determine the best correlation with true tissue deformations. In such a way we may be able to predict certain properties of the apical layer in organisms undergoing this type of morphogenetic change.

\subsection{Apical constriction in a spherical shell}

Another geometry in which apical constriction is physiologically-relevant is that of a spherical shell, which may be used to model the initial invaginations of the gastrulation process. In this case we assume spherical symmetry (i.e. all quantities are independent of the azimuthal angle $\phi$, and the azimuthal displacement is zero). We assume an isotropic contraction $\eta_{\alpha \beta}=$ $f(\theta) g_{\alpha \beta}$, where $f(\theta)$ is of the same form as for the cylinder and plate, but centred on the north pole of the sphere. A Winkler foundation is again prescribed. The
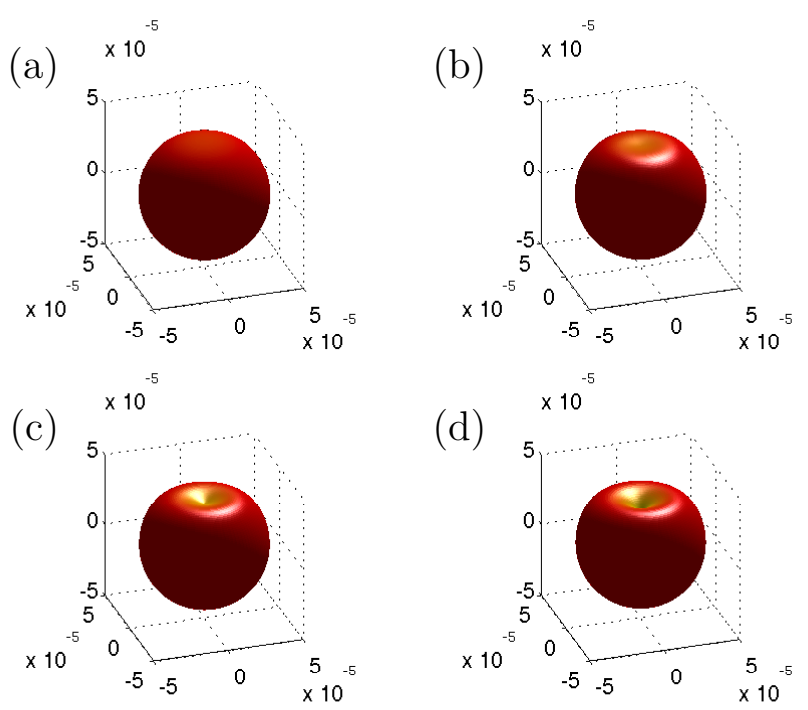

Figure 11 Increased bending in a spherical shell due to apical constriction. The surfaces (a)-(d) represent $\tilde{f}$ increasing through $0.15,0.3,0.45,0.6$ respectively. Colour is related to $f(x)$ as for Figure 9.

numerical results from Matlab are shown in Figure 11; once again the equations are omitted for lack of space. We can see that as the degree of constriction increases, the shell first flattens at the pole, then an increasing amount of invagination is evident, in accordance with observations of invaginating embryos (as can be seen by comparison with Figure 1). Once again this is but one numerical calculation; further calculations should be compared with actual data in order to glean some information regarding the apical constriction process by comparing theory with experiment.

\section{Conclusions}

We have succeeded in developing a thin elastic shell model that incorporates the apical constriction mechanism responsible for tissue bending in embryonic epithelial tissues. We have applied the theory to certain simple geometries to model some of the critical tissue folding events in embryonic development, such as the initial tissue bending in neurulation, ventral furrow formation, and gastrulation. The results, as displayed in Figures 9-11, qualitatively agree with experimental images of embryos.

The theory developed here may be extended to more complicated geometries; the equations written at the end of Section 3.1 hold for any geometry, with the proviso that the equations become more complicated as the geometry becomes less symmetric. In particular, the examples in Figures 9-11 were calculated by ordinary 
differential equations (ODEs) due to axisymmetry (for the sphere) or invariance in one direction (for the plate and cylinder). Morphogenetic events without this sort of symmetry would require a partial differential equation method to solve them, such as a finite element method. The equations of Section 3.1 describe the deformation of an anisotropic inhomogeneous shell with given surface tractions, and so any finite element package able to model such shell problems will be able to model morphogenesis as described by the theory of Section 3.1.

Another aspect of the morphogenetic folding that was simplified in the numerical experiments of Section 4 was the exact nature of the fibres' contraction. We imposed a given contraction that was independent of all other physical parameters of the system. While this seems to be a common approach in the modelling literature - since the mechanism by which the fibres contract is still a mystery — some, especially Odell et al (1981) gave the fibres a more active role, by assuming that the tension transmitted by the apical region was linked to its compression through a given ODE. Such an approach could be incorporated into our theory by making the material length of the fibres (represented by $\eta_{\alpha \beta}$ ) dependent on the current deformation state (governed by $\gamma_{\alpha \beta}$ and $\rho_{\alpha \beta}$ ). Our theory also allows the constriction to be governed by the relative concentrations of diffusing morphogens.

The theory as presented, however, does have limitations, due to some of the simplifying and constitutive assumptions made in its derivation. Among these is the assumption of elasticity for the epithelial tissue. One of the key drivers of morphogenetic deformations is the rearranging of cells, and this is discounted by our theory. However, this should not detract from the theory's validity as long as we recognise that only the initial stages of the folding are modelled by this theory. A possible extension to this work could therefore be the inclusion of plasticity into the model to account for cell rearrangements.

Another class of limitations of this theory is the number of simplifying assumptions made within the elastic shell theory: for example, the use of linear shell theory, and the assumption of linearity in the response of the fibres to deformation. These assumptions and others may be relaxed at the expense of a much more complicated model than that derived in Section 3.1; it is not clear however that such a relaxation would yield more physically-realistic tissue behaviour.

Acknowledgements The authors acknowledge funding from the Engineering and Physical Sciences Research Council as part of the "New Frontiers in the Mathematics of Solids" (OxMOS) programme EP/D048400/1.

Figure 1 is reprinted from Methods in Cell Biology, Vol. 27, John B. Morrill, Scanning Electron Microscopy of Embryos, Pages 263-293, Copyright (1986), with permission from Elsevier. We thank the reviewers for their helpful comments.

\section{References}

Bement WM, Mooseker MS (1996) The cytoskeleton of the intestinal epithelium: components, assembly, and dynamic rearrangements. In: Hesketh JE, Pryme IF (eds) The Cytoskeleton: A Multi-Volume Treatise. Volume 3: Cytoskeleton in Specialized Tissues and in Pathological States, Elsevier, pp 359-404, DOI 10.1016/S1874-6020(96)80015-2

Brodland GW, Wiebe CJ (2004) Mechanical effects of cell anisotropy on epithelia. Computer Methods in Biomechanics and Biomedical Engineering 7(2):9199, DOI 10.1080/1025584042000209369

Burnside B (1971) Microtubules and microfilaments in newt neurulation. Developmental Biology 26(3):416441, DOI 10.1016/0012-1606(71)90073-X

Clausi DA, Brodland GW (1993) Mechanical evaluation of theories of neurulation using computer simulations. Development 118(3):1013-1023

Cummings FW (1990) A model of morphogenetic pattern formation. Journal of Theoretical Biology 144(4):547-566, DOI 10.1016/S0022-5193(05)80089$\mathrm{X}$

Davidson LA (2008) Integrating morphogenesis with underlying mechanics and cell biology. Current Topics in Developmental Biology 81:113-133, DOI 10.1016/S0070-2153(07)81003-9

Davidson LA, Koehl MAR, Keller R, Oster GF (1995) How do sea urchins invaginate? Using biomechanics to distinguish between mechanisms of primary invagination. Development 121(7):2005-2018

Davidson LA, Oster GF, Keller RE, Koehl MAR (1999) Measurements of mechanical properties of the blastula wall reveal which hypothesized mechanisms of primary invagination are physically plausible in the sea urchin Strongylocentrotus purpuratus. Developmental Biology 209(2):221-238, DOI 10.1006/dbio.1999.9249

Dawes-Hoang RE, Parmar KM, Christiansen AE, Phelps CB, Brand AH, Wieschaus EF (2005) Folded gastrulation, cell shape change and the control of myosin localization. Development 132(18):41654178, DOI 10.1242/dev.01938

Dervaux J, Ciarletta P, Ben Amar M (2009) Morphogenesis of thin hyperelastic plates: a constitutive theory of biological growth in the Föppl-von Kármán 
limit. Journal of the Mechanics and Physics of Solids 57(3):458-471, DOI 10.1016/j.jmps.2008.11.011

Fristrom D (1988) The cellular basis of epithelial morphogenesis. A review. Tissue and Cell 20(5):645-690, DOI 10.1016/0040-8166(88)90015-8

Gierer A (1977) Physical aspects of tissue evagination and biological form. Quarterly Reviews of Biophysics 10(4):529-593, DOI 10.1017/S0033583500003218

Gilbert SF (2006) Developmental Biology, eighth edn. Sinauer Associates, Sunderland, Massachusetts

Gordon R, Brodland GW (1987) The cytoskeletal mechanics of brain morphogenesis. Cell Biochemistry and Biophysics 11(1):177-238

Graebert KS, Bauch H, Neumüller W, Brix K, Herzog V (1997) Epithelial folding in vitro: studies on the cellular mechanism underlying evagination of thyrocyte monolayers. Experimental Cell Research 231(1):214225, DOI 10.1006/excr.1996.3456

Hardin J, Keller R (1988) The behaviour and function of bottle cells during gastrulation of Xenopus laevis. Development 103(1):211-230

Hardin JD, Cheng LY (1986) The mechanisms and mechanics of archenteron elongation during sea urchin gastrulation. Developmental Biology 115(2):490-501, DOI 10.1016/0012-1606(86)90269-1

Hutson MS, Ma X (2008) Mechanical aspects of developmental biology: perspectives On Growth and Form in the (post)-genomic age. Physical Biology 5(1):015,001, DOI 10.1088/1478-3975/5/1/015001

Jones GW, Chapman SJ (2009) Modelling growth in biological materials. SIAM Review (submitted)

Keller R, Davidson LA, Shook DR (2003) How we are shaped: the biomechanics of gastrulation. Differentiation 71(3):171-205, DOI 10.1046/j.14320436.2003.710301.x

Koiter WT (1966) On the nonlinear theory of thin elastic shells. Proceedings of the Koninklijke Nederlandse Akademie van Wetenschappen (Royal Dutch Academy of Sciences), Ser B 69:1-54

Lanir Y (1983) Constitutive equations for fibrous connective tissues. Journal of Biomechanics 16(1):1-12, DOI 10.1016/0021-9290(83)90041-6

Lee JY, Harland RM (2007) Actomyosin contractility and microtubules drive apical constriction in Xenopus bottle cells. Developmental Biology 311(1):4052, DOI 10.1016/j.ydbio.2007.08.010

Lewis WH (1947) Mechanics of invagination. The Anatomical Record 97(2):139-156, DOI 10.1002/ar.1090970203

Lim CT, Zhou EH, Quek ST (2006) Mechanical models for living cells - a review. Journal of Biomechanics 39(2):195-216, DOI 10.1016/j.jbiomech.2004.12.008
Martin AC, Kaschube M, Wieschaus EF (2009) Pulsed contractions of an actin-myosin network drive apical constriction. Nature 457(7228):495-499, DOI 10.1038/nature07522

Mittenthal JE (1987) The shaping of cell sheets: an application of mechanics in developmental biology. In: Akkas N (ed) Biomechanics of Cell Division, NATO ASI Series A: Life Sciences, vol 132, Plenum Press, New York, pp 327-346

Mittenthal JE, Mazo RM (1983) A model for shape generation by strain and cell-cell adhesion in the epithelium of an arthropod leg segment. Journal of Theoretical Biology 100(3):443-483, DOI 10.1016/00225193(83)90441-1

Morrill JB (1986) Scanning electron microscopy of embryos, Methods in Cell Biology, vol 27, Elsevier, chap 15, pp 263-293. DOI 10.1016/S0091679X(08)60353-2

Muñoz JJ, Barrett K, Miodownik M (2007) A deformation gradient decomposition method for the analysis of the mechanics of morphogenesis. Journal of Biomechanics 40(6):1372-1380, DOI 10.1016/j.jbiomech.2006.05.006

Niordson FI (1985) Shell Theory. North-Holland, Amsterdam

Odell GM, Oster G, Alberch P, Burnside B (1981) The mechanical basis of morphogenesis. I. Epithelial folding and invagination. Developmental Biology 85(2):446-462, DOI 10.1016/0012-1606(81)90276-1

Pilot F, Lecuit T (2005) Compartmentalized morphogenesis in epithelia: from cell to tissue shape. Developmental Dynamics 232(3):685-694, DOI $10.1002 /$ dvdy.20334

Ramasubramanian A, Taber LA (2008) Computational modeling of morphogenesis regulated by mechanical feedback. Biomechanics and Modeling in Mechanobiology 7(2):77-91, DOI 10.1007/s10237-007-0077-y

Schöck F, Perrimon N (2002) Molecular mechanisms of epithelial morphogenesis. Annual Review of Cell and Developmental Biology 18:463-493, DOI 10.1146/annurev.cellbio.18.022602.131838

Stern CD (2004) Gastrulation: From Cells to Embryos. Cold Spring Harbor Laboratories Press, New York

Suiker ASJ, Chang CS (2000) Application of higherorder tensor theory for formulating enhanced continuum models. Acta Mechanica 142(1-4):223-234, DOI 10.1007/BF01190020

Sweeton D, Parks S, Costa M, Wieschaus E (1991) Gastrulation in Drosophila: the formation of the ventral furrow and posterior midgut invaginations. Development 112(3):775-789

Thomas GH (2001) Spectrin: the ghost in the machine. BioEssays 23(2):152-160, DOI 10.1002/1521- 
1878(200102)23:2<152::AID-BIES1022>3.0.CO;2-1

Wiebe C, Brodland GW (2005) Tensile properties of embryonic epithelia measured using a novel instrument. Journal of Biomechanics 38(10):2087-2094, DOI 10.1016/j.jbiomech.2004.09.005

Wright NA, Alison M (1984) The Biology of Epithelial Cell Populations. Oxford University Press

Zinemanas D, Nir A (1992) A fluid-mechanical model of deformation during embryo exogastrulation. Journal of Biomechanics 25(4):341-346, DOI 10.1016/00219290(92)90253-W 